\title{
Holographic Floquet states in low dimensions
}

\author{
Ana Garbayo, Javier Mas and Alfonso V. Ramallo \\ Departamento de Física de Partículas, Universidade de Santiago de Compostela, \\ and Instituto Galego de Física de Altas Enerxías (IGFAE), \\ E-15782 Santiago de Compostela, Spain \\ E-mail: ana.garbayo.peon@usc.es, javier.mas@usc.es, \\ alfonso@fpaxp1.usc.es
}

ABSTRACT: We study the response of a $(2+1)$-dimensional gauge theory to an external rotating electric field. In the strong coupling regime such system is formulated holographically in a top-down model constructed by intersecting D3- and D5-branes along $2+1$ dimensions, in the quenched approximation, in which the D5-brane is a probe in the $A d S_{5} \times \mathbb{S}^{5}$ geometry. The system has a non-equilibrium phase diagram with conductive and insulator phases. The external driving induces a rotating current due to vacuum polarization (in the insulator phase) and to Schwinger effect (in the conductive phase). For some particular values of the driving frequency the external field resonates with the vector mesons of the model and a rotating current can be produced even in the limit of vanishing driving field. These features are in common with the $(3+1)$ dimensional setup based on the D3-D7 brane model $[26,27]$ and hint on some interesting universality. We also compute the conductivities paying special attention to the photovoltaic induced Hall effect, which is only present for massive charged carriers. In the vicinity of the Floquet condensate the optical Hall coefficient persists at zero driving field, signalling time reversal symmetry breaking.

KEYwords: AdS-CFT Correspondence, D-branes

ArXiv EPrint: 2007.12115 


\section{Contents}

1 Introduction $\quad 2$

2 Setup and ansatz $\quad 4$

$\begin{array}{lll}2.1 & \text { Boundary conditions } & 7\end{array}$

3 Types of embeddings 8

3.1 Black hole embeddings 8

3.2 Minkowski embeddings 9

3.3 Critical embeddings 9

$\begin{array}{lll}3.4 & \text { Effective metric } & 10\end{array}$

4 Phase diagram 11

5 Analytic solutions $\quad \mathbf{1 5}$

$\begin{array}{lll}5.1 & \text { Massless embeddings } & 15\end{array}$

$\begin{array}{ll}5.2 \text { High frequency limit } & 17\end{array}$

6 Conductivities 18

6.1 AC conductivities 22

6.2 DC conductivities 24

$\begin{array}{ll}6.3 \text { Massless limit } & 27\end{array}$

$\begin{array}{lll}7 & \text { Summary and outlook } & 27\end{array}$

$\begin{array}{lr}\text { A Regularity conditions } & 29\end{array}$

A.1 Large frequency 30

$\begin{array}{lll}\text { A.2 Small frequency } & 30\end{array}$

B Small mass solutions $\quad 30$

C Embeddings in the linearized approximation 33

C.1 Minkowski embeddings 33

C.2 Black hole embeddings 35

$\begin{array}{lll}\text { C.3 Critical embeddings } & 38\end{array}$

D Conductivities in the massless case $\quad 39$

E More on optical conductivities $\quad 42$ 


\section{Introduction}

The physics of periodically driven quantum systems has been the subject of intense study in recent years (see [1-4] for reviews with tons of citations). Several reasons back up this interest, a very relevant one being technological in origin: the possibility of manipulating quantum systems in a controlled way by using time-periodic external fields. This approach goes under the name of Floquet engineering [5, 6], following from the Floquet theorem, a temporal analogue of the Bloch theorem. The artificial setup involves mainly irradiating the system with a circularly polarised laser, or shaking it around. With appropriate periodic drivings, new phases of quantum materials have been created and non-equilibrium phenomena have emerged. Examples include light-induced superconductivity $[7,8]$ and Floquet topological insulators [9, 11, 13-17]. Moving on to $(3+1)$ dimensions, artificial Weyl semimetals have also been reported [18-20].

In this paper we study the behavior of a strongly-coupled $(2+1)$-dimensional gauge theory under the influence of a external electric field $\overrightarrow{\mathcal{E}}$ rotating in the $x y$ plane as

$$
\mathcal{E}_{x}+i \mathcal{E}_{y}=E e^{i \Omega t} .
$$

We will address this problem in the context of the holographic AdS/CFT correspondence [21] (see [22-25] for reviews), and will follow closely the pioneering work in refs. $[26,27]$ in their study of $(3+1)$-dimensional systems. In the first of these two references, the authors studied the massless D3-D7 flavour system and computed the artificially induced Hall conductivity. In the second they extend part of the analysis to massive flavours where an interesting phase space shows up. Our paper streamlines the same analysis in $(2+1)$ dimensions, extending the analysis to encompass the optical and static conductivities also for massive flavours. The $(2+1)$-dimensional theory in place is realised as a codimension-one defect in $\mathcal{N}=4$ supersymmetric $\mathrm{SU}\left(N_{c}\right)$ super Yang-Mills theory in four dimensions. The field content and lagrangian was explicitly constructed in [28]. It contains a matter hypermultiplet living in the defect which couples to the fields of the ambient four-dimensional theory. In String Theory, this model can be realized as the intersection of D3- and D5-branes along $2+1$ dimensions. In this setup the $4 \mathrm{~d}$ gauge theory on the D3-branes is holographically dual to the $A d S_{5} \times \mathbb{S}^{5}$ geometry, while the D5-branes provide $(2+1)$-dimensional flavors, i.e., fields living in the fundamental representation of the gauge group [29-31]. We will work in the quenched probe approximation and will hence neglect the backreaction of the D5-branes on the $A d S_{5} \times \mathbb{S}^{5}$ geometry. The fluctuation of the fields living on the brane are dual to the mesonic excitations of the gauge theory. In ref. [32] the complete analysis of these fluctations was performed for the D3-D5 system and the exact spectrum of mesons was found (see [34] for a similar analysis in the D3-D7 case).

When an external electric field acts on a medium, a vacuum polarization due to virtual charged particles (quarks) is produced. As a consequence, an oscillating polarization current is induced. If the external field is weak enough, the medium will remain in an insulator gapped phase in which the induced current is always perpendicular to the applied field. There is no Joule heating and the system is dissipation-less. When the external field is large enough the vacuum is unstable against the creation of quark-antiquark pairs (Schwinger 
effect). In the conductive phase the current becomes dissipative, and the driving induces a Joule heating.

For an external oscillating electric field as in (1.1) the critical value $E_{c}$ at which the insulator-conductor transition takes place depends on the frequency $\Omega$, i.e., $E_{c}=$ $E_{c}(\Omega)$ [33]. Remarkably, for some values $\Omega=\Omega_{c}$ of the frequency, the transition occurs for vanishing external electric field, i.e., $E_{c}\left(\Omega_{c}\right)=0$. At this point of the phase space the rotating current $j$ is not zero, in spite of the fact that the external driving field vanishes. Physically, for these frequencies the driving field enters in resonance with the vector meson excitations of the gauge theory. Actually, this resonating states with zero electric field and non-zero current also occur in the conductive pase in a finite range of frequencies $\Omega_{c}<\Omega \leq \Omega_{m}$. Following [27], where this behaviour was first found in the D3-D7 model, we will call a configuration with $E=0$ and $j \neq 0$ a zero field Floquet condensate of vector mesons, or simply a vector meson Floquet condensate. Such stable rotating state is a fixed point of time evolution, namely a non-thermal fixed point [35].

In our holographic setup the flavor degrees of freedom are modeled as the excitations of a probe D5-brane embedded in the $A d S_{5} \times \mathbb{S}^{5}$ ten-dimensional background. The probe brane extends along an $A d S_{4} \times \mathbb{S}^{2}$ submanifold of the ten-dimensional background. The holographic duality in the D5-brane worldvolume relates the fields at the boundary of $A d S_{4}$ to those of the dual $(2+1)$-dimensional gauge theory. In particular, to model the rotating field (1.1) we have to switch on an electric gauge field on the worldvolume attaining the value (1.1) as we approach the $A d S_{4}$ boundary (the subleading term near the boundary determines the current $j$ ). The D5-brane with this world-volume gauge field has a nontrivial profile which can be found by solving the equations of motion of the probe brane. The leading value of the profile function at the boundary determines mass of the quarks, while the subleading term is related to the quark condensate.

A probe brane with an electric field in his worldvolume can develop an event horizon in the effective (open string) metric on its worldvolume. This is analogous, but not the same, to the case in which the brane is embedded in a black hole background geometry with a non-zero Hawking temperature [37-39]. Accordingly, we can borrow the terminology of the non-zero temperature case and classify the configurations of the brane depending on whether or not it crosses or not the effective horizon. In the so-called Minkowski embeddings the brane reaches the origin of $A d S_{4}$ without developing the effective horizon. These Minkowski configurations are dual to the insulating phase of the defect gauge theory. On the contrary, in the conductive phase the induced horizon forms at a finite radial distance from the D3-brane system. The flavour mesons are deconfined and a current can set up in response to an external electric field. At the interface between these two cases we find the critical embeddings, in which the probe develops the effective horizon right at its IR endpoint. In this work we study both types of embeddings in order to determine the stable non-equilibrium phases of the theory. In this task we will employ both numerical and analytic techniques.

The rest of this paper is organized as follows. In section 2 we present our setup, find the equations of motion for the probe D5-brane and study the boundary behavior of the different functions and their relation to the observables of the gauge theory. In section 3 we 
analyze the different types of embeddings and determine the IR boundary conditions that regular solutions must satisfy. We also determine in this section the effective metric and the effective Hawking temperature. The numerical integration of the equations of motion and the structure of the phase diagram of the theory is presented in section 4 .

When either the mass is zero or the driving frequency is large, the equations of motion can be solved analytically in the linearized approximation. The exact analytical solutions are presented in section 5. Section 6 deals with the analysis of the AC and DC conductivities of the model, which are obtained by studying the response of the brane to an additional probe electric field which is treated in a linearized approximation. We end up with a summary and some concluding remarks in section 7 . The paper is supplemented with some appendices. In appendix A we give details about the derivation of the regularity conditions of the brane embeddings at the pseudo-horizon. In appendix B, we find approximate analytic solutions of the equations of motion when the mass is small. The different embeddings when the equations of motion are linearized are studied in appendix C. The exact conductivities for the massless case are obtained in appendix D.

\section{Setup and ansatz}

In order to analyze the Floquet states in the strong coupling regime, we will engineer a holographic brane setup in which two sets of branes intersect along $2+1$ dimensions. More concretely we will consider the intersection of two stacks of D3- and D5-branes according to the following array

$$
\begin{array}{lllllllll}
1 & 2 & 3 & 4 & 5 & 6 & 7 & 8 & 9
\end{array}
$$

$$
\begin{aligned}
& D 3: \times \times \times \ldots+\ldots \\
& D 5: \times \times \ldots \times \times \ldots
\end{aligned}
$$

In (2.1) the D3- and D5-branes share the spatial directions 1 and 2, whereas the direction 3 is parallel to the D3-brane and orthogonal to the D5-brane. The field theory dual to the brane setup (2.1) is well-known [28-31]. It consists of a supersymmetric theory with $N_{f}$ matter hypermultiplets (flavors) living on the $(2+1)$-dimensional defect and coupled to the ambient $\mathcal{N}=4$ theory realized by the stack of $N_{c}$ color D3-branes. We will adopt the approximation in which the D5-branes are probes in the geometry generated by the D3-branes, so $N_{f} \ll N_{c}$. The latter is the standard holographic dual of four-dimensional $\mathcal{N}=4$ super Yang-Mills, namely $A d S_{5} \times \mathbb{S}^{5}$ with flux. Let us start by recalling this geometry

$$
\begin{aligned}
d s_{10}^{2}= & \frac{\rho^{2}+w_{1}^{2}+w_{2}^{2}+w_{3}^{2}}{R^{2}}\left(-d t^{2}+d x^{2}+d y^{2}+d z^{2}\right)+ \\
& +\frac{R^{2}}{\rho^{2}+w_{1}^{2}+w_{2}^{2}+w_{3}^{2}}\left(d \rho^{2}+\rho^{2} d \Omega_{2}^{2}+d w_{1}^{2}+d w_{2}^{2}+d w_{3}^{2}\right),
\end{aligned}
$$

where $d \Omega_{2}^{2}=d \theta^{2}+\sin ^{2} \theta d \phi^{2}$ is the metric of a unit two-sphere and $R$ is the common radius of the $A d S_{5}$ and $\mathbb{S}^{5}$ factors (in what follows we will take $R=1$ ). In order to embed a D5-brane wrapping a $A d S_{4} \times \mathbb{S}^{2}$ submanifold we choose the following set of worldvolume 
coordinates

$$
\xi^{a}=(t, x, y, \rho, \theta, \phi),
$$

and the following ansatz for the transverse scalars

$$
z=\text { constant }, \quad w_{1}=w(t, \rho), \quad w_{2}=w_{3}=0 .
$$

The induced metric on the D5-brane woldvolume now takes the form

$$
\begin{aligned}
d s_{6}^{2}= & -\left(\rho^{2}+w^{2}\right)\left(1-\frac{\dot{w}^{2}}{\left(\rho^{2}+w^{2}\right)^{2}}\right) d t^{2}+\left(\rho^{2}+w^{2}\right)\left(d x^{2}+d y^{2}\right)+ \\
& +\frac{1}{\rho^{2}+w^{2}}\left(\left(1+w^{2}\right) d \rho^{2}+2 \dot{w} w^{\prime} d t d \rho+\rho^{2} d \Omega_{2}^{2}\right)
\end{aligned}
$$

where the dot denotes derivative with respect to $t$ and the prime with respect to the holographic coordinate $\rho$. The main objective to study the response of this system to the driving of an external circularly polarized electric fied

$$
\overrightarrow{\mathcal{E}}(t)=\left(\begin{array}{c}
\mathcal{E}_{x}(t) \\
\mathcal{E}_{y}(t)
\end{array}\right)=\left(\begin{array}{cc}
\cos \Omega t & -\sin \Omega t \\
\sin \Omega t & \cos \Omega t
\end{array}\right)\left(\begin{array}{c}
E_{x} \\
E_{y}
\end{array}\right) \equiv O(t) \vec{E},
$$

with $\vec{E}=\overrightarrow{\mathcal{E}}(t=0)$. This will source of a worldvolume gauge field with one-form potential

$$
2 \pi \alpha^{\prime} \mathcal{A}=a_{x}(t, \rho) d x+a_{y}(t, \rho) d y
$$

whose field strength is

$$
2 \pi \alpha^{\prime} \mathcal{F}=\dot{a}_{x} d t \wedge d x+a_{x}^{\prime} d \rho \wedge d x+\dot{a}_{y} d t \wedge d y+a_{y}^{\prime} d \rho \wedge d y .
$$

The action of the probe brane is given by the DBI action

$$
S=-N_{f} T_{5} \int d^{6} \xi \sqrt{-\operatorname{det}\left(g_{6}+2 \pi \alpha^{\prime} \mathcal{F}\right)},
$$

where $T_{5}$ is the tension of the D5-brane. In the following we will adhere to the notation and reasoning advocated in [26, 27], with slight modifications. To start with, the following switch from vector to complex notation is useful

$$
E=E_{x}+i E_{y}, \quad a=a_{x}+i a_{y} .
$$

Now, computing the determinant $-\operatorname{det}\left(g_{6}+2 \pi \alpha^{\prime} F\right)$, the DBI action becomes

$$
\begin{aligned}
S \sim & -\int d t d \rho \frac{\rho^{2}}{\rho^{2}+w^{2}}\left[\left(1+w^{\prime 2}\right)\left(\left(\rho^{2}+w^{2}\right)^{2}-|\dot{a}|^{2}\right)-\dot{w}^{2}\left(1+\left|a^{\prime}\right|^{2}\right)+\right. \\
& +\left|a^{\prime}\right|^{2}\left(\rho^{2}+w^{2}\right)^{2}+2 \dot{w} w^{\prime}\left(\operatorname{Re}\left(\dot{a} a^{\prime *}\right)-\left(\operatorname{Im}\left(\dot{a} a^{\prime *}\right)\right)^{2}\right]^{\frac{1}{2}} .
\end{aligned}
$$

Another convenient switch is to represent the complex gauge field $a$ in the rotating frame

$$
\mathcal{E}=E e^{i \Omega t}, \quad a(t, \rho)=b(t, \rho) e^{i(\Omega t+\chi(t, \rho))} .
$$


In the new variables, $(b, \chi)$ the DBI action $(2.11)$ becomes

$$
\begin{aligned}
S \sim & -\int d t d \rho \frac{\rho^{2}}{\rho^{2}+w^{2}}\left[\left(1+w^{2}\right)\left(\left(\rho^{2}+w^{2}\right)^{2}-\dot{b}^{2}-(\Omega+\dot{\chi})^{2} b^{2}\right)-\right. \\
& -\dot{w}^{2}\left(1+b^{\prime 2}+b^{2} \chi^{\prime 2}\right)+\left(\rho^{2}+w^{2}\right)^{2}\left(b^{\prime 2}+b^{2} \chi^{\prime 2}\right)+ \\
& \left.+2 \dot{w} w^{\prime}\left(\dot{b} b^{\prime}+(\Omega+\dot{\chi}) \chi^{\prime} b^{2}\right)-b^{2}\left(\dot{b} \chi^{\prime}-(\Omega+\dot{\chi}) b^{\prime}\right)\right]^{\frac{1}{2}} .
\end{aligned}
$$

Since the action does not depend on $t$ explicitly, taking the functions $b, \chi$ and $w$ as independent of the time is a consistent ansatz ${ }^{1}$

$$
b=b(\rho), \quad \chi=\chi(\rho), \quad w=w(\rho) .
$$

By removing the terms with the time derivative from the action, we can rewrite it in a much more simplified fashion as $S \sim \int d \rho \mathcal{L}$, where now

$$
\mathcal{L}=\frac{\rho^{2}}{\rho^{2}+w^{2}} \sqrt{\left(\left(\rho^{2}+w^{2}\right)^{2}-\Omega^{2} b^{2}\right)\left(1+b^{\prime 2}+w^{\prime 2}\right)+\left(\rho^{2}+w^{2}\right)^{2} b^{2} \chi^{\prime 2}} .
$$

We first notice that $\chi$ is a cyclic variable which means that $q$, defined as

$$
q=\Omega \frac{\partial \mathcal{L}}{\partial \chi^{\prime}}=\Omega \frac{\rho^{4}}{\mathcal{L}} b^{2} \chi^{\prime},
$$

is independent of $\rho$. The Euler-Lagrange equations derived from $\mathcal{L}$ are

$$
\begin{aligned}
& \rho\left(\rho^{2}+w^{2}\right)\left[\left(\rho^{2}+w^{2}\right)^{2}-\Omega^{2} b^{2}\right] b^{\prime \prime}= \\
&=-\left(1+b^{\prime 2}+w^{\prime 2}\right)\left[2\left(\rho^{2}+w^{2}\right)^{3} b^{\prime}+\Omega^{2} b\left(\rho\left(\rho^{2}+w^{2}\right)-2 w^{2} b b^{\prime}\right)\right]+ \\
&+b\left(\rho^{2}+w^{2}\right)^{3}\left(\rho-2 b b^{\prime}\right) \chi^{\prime 2}, \\
& \rho\left(\rho^{2}+w^{2}\right)\left[\left(\rho^{2}+w^{2}\right)^{2}-\Omega^{2} b^{2}\right] w^{\prime \prime}= \\
& \quad-2\left(1+b^{\prime 2}+w^{\prime 2}\right)\left[\left(\rho^{2}+w^{2}\right)^{3} w^{\prime}-\Omega^{2} b^{2} w\left(\rho+w w^{\prime}\right)\right]-2 b^{2}\left(\rho^{2}+w^{2}\right)^{3} w^{\prime} \chi^{\prime 2}, \\
& b \rho\left(\rho^{2}+w^{2}\right)\left[\left(\rho^{2}+w^{2}\right)^{2}-\Omega^{2} b^{2}\right] \chi^{\prime \prime}= \\
& \quad-2 \chi^{\prime}\left[\left(\rho^{2}+w^{2}\right)^{3}\left(b^{3} \chi^{\prime 2}+\rho b^{\prime}+b\left(1+b^{\prime 2}+w^{\prime 2}\right)\right)-\right. \\
&\left.\quad-\Omega^{2} b^{3}\left(2 \rho^{2}+2 \rho w w^{\prime}+w^{2}\left(1+b^{\prime 2}+w^{\prime 2}\right)\right)\right] .
\end{aligned}
$$

The last equation in (2.17) is redundant, since we can use the first integral (2.16) to obtain the value of $\chi^{\prime}$ in terms of the other functions

$$
\chi^{\prime 2}=\frac{q^{2}\left[\left(\rho^{2}+w^{2}\right)^{2}-\Omega^{2} b^{2}\right]}{\left(\rho^{2}+w^{2}\right)^{2} b^{2}\left[\Omega^{2} \rho^{4} b^{2}-q^{2}\right]}\left(1+b^{\prime 2}+w^{\prime 2}\right) .
$$

We can next use this last equation to eliminate $\chi^{\prime}$ in the first two equations in (2.17). The resulting equations for $w$ and $b$ depend on $q$ (alternatively one could Legendre transform and obtain the corresponding Routhian).

\footnotetext{
${ }^{1}$ This of course does not preclude the existence of time dependent solutions. For example, at special points in phase space, instabilities may trigger first order phase transitions that can occur between Black Hole and Minkowski embeddings.
} 


\subsection{Boundary conditions}

Let us now study the equations of motion of the gauge field at the UV $\rho \rightarrow \infty$. It is convenient to define the new complex combination

$$
c(\rho)=b(\rho) e^{i \chi(\rho)},
$$

hence $a(\rho, t)=c(\rho) e^{i \Omega t}$. In terms of $c(\rho)$ the lagrangian density $\mathcal{L}$ gives

$$
\mathcal{L} \sim \frac{\rho^{2}}{\rho^{2}+w^{2}}\left[\left(1+w^{\prime 2}\right)\left[\left(\rho^{2}+w^{2}\right)^{2}-\Omega^{2}|c|^{2}\right]+\left(\rho^{2}+w^{2}\right)^{2}\left|c^{\prime}\right|^{2}-\Omega^{2}\left[\operatorname{Re}\left(c c^{\prime *}\right)\right]^{2}\right]^{\frac{1}{2}},
$$

and the equation of motion for $c(\rho)$ derived from $\mathcal{L}$ is

$$
\begin{aligned}
& \partial_{\rho}\left[\frac{\rho^{4}}{\left(\rho^{2}+w^{2}\right)^{2} \mathcal{L}}\left(\left(\rho^{2}+w^{2}\right)^{2} c^{\prime}-\Omega^{2} \operatorname{Re}\left(c c^{*}\right) c\right)\right]+ \\
& \quad+\Omega^{2} \frac{\rho^{4}}{\left(\rho^{2}+w^{2}\right)^{2} \mathcal{L}}\left(\left(1+w^{\prime 2}\right) c+\operatorname{Re}\left(c c^{\prime *}\right) c^{\prime}\right)=0 .
\end{aligned}
$$

Near the UV at $\rho \rightarrow \infty$ we can show that $\mathcal{L} \approx \rho^{2}$ and the equation of motion reduces to $\partial_{\rho}\left(\rho^{2} c^{\prime}\right)=0$, whose general solution is of the form $c(\rho)=a_{1}+a_{2} / \rho$. Redefining the constants $a_{1}$ and $a_{2}$, we can write asymptotically

$$
c(\rho)=b(\rho) e^{i \chi(\rho)}=\frac{i E}{\Omega}+\frac{j}{\rho}+\cdots,
$$

where $E$ is the complexified electric field defined in (2.12), and $j$ the related current. Proceeding similarly, the embedding function $w(\rho)$ behaves near the UV boundary $\rho \rightarrow$ $\infty$ as

$$
w(\rho) \sim m+\frac{\mathcal{C}}{\rho}+\cdots,
$$

where $m$ and $\mathcal{C}$ are constants related to the quark mass and quark condensate respectively. We will actually refer to them as such. The relation between $E, j, m$ and $\mathcal{C}$ and the electric field $\mathcal{E}_{\mathrm{YM}}(t)$, the electric current $\mathcal{J}_{\mathrm{YM}}(t)$, quark mass $m_{q}$ and quark condensate $\left\langle O_{m}\right\rangle$ in the boundary theory are

$$
\begin{aligned}
\mathcal{E}_{\mathrm{YM}}(t) & =\sqrt{\frac{\lambda}{2 \pi^{2}}} e^{i \Omega t} E, & \mathcal{J}_{\mathrm{YM}}(t) & =\frac{N_{f} N_{c}}{\pi^{2}} e^{i \Omega t} j, \\
m_{q} & =\sqrt{\frac{\lambda}{2 \pi^{2}}} m, & \left\langle O_{m}\right\rangle & =-\frac{N_{f} N_{c}}{\pi^{2}} c,
\end{aligned}
$$

where $\lambda=g_{\text {YM }}^{2} N_{c}$ is the 't Hooft coupling of the $\mathcal{N}=4$ theory. The integration constant $q$ defined in (2.16) can be written in terms of the field $c$ and $c^{*}$

$$
q=i \frac{\Omega \rho^{4}}{2 \mathcal{L}}\left(c c^{*}-c^{*} c^{\prime}\right),
$$

and can be easily related to UV data. As $w(\rho) \sim m+\mathcal{C} / \rho$ when $\rho \rightarrow \infty$, it follows that $\mathcal{L} \approx \rho^{2}$ for large $\rho$. Moreover, from (2.22) $c^{\prime} \sim-j / \rho^{2}$ and, therefore

$$
q=\operatorname{Re}\left(E j^{*}\right)=j_{x} E_{x}+j_{y} E_{y},
$$


which endows $q$ with the physical interpretation of the Joule heating. If we assume that the driving effectively heats the system we should expect that $q \geq 0$, and from (2.16) this implies $\Omega \chi^{\prime} \geq 0$. We can see that this result is consistent with the ingoing boundary condition at the pseudohorizon, which can be argued as follows. The wavefront of the complexified gauge potential $a(t, \rho)$ in $(2.12)$ is the surface $\chi(\rho)+\Omega t=$ constant (we are in the case in which $\chi$ does not depend on $t$ ). By performing a generic variation of this condition, it follows that

$$
\delta \rho=-\frac{\Omega}{\chi^{\prime}} \delta t .
$$

Requiring that our gauge potential represents an infalling wave at the pseudo-horizon implies $\delta \rho<0$ for $\delta t>0$ which, following (2.28), only occurs when $\Omega \chi^{\prime} \geq 0$ and, therefore, $q \geq 0$.

The equations of motion (2.17) and the UV boundary conditions (2.22) and (2.23) have the following scaling symmetry

$$
\begin{aligned}
& t \rightarrow t / \alpha, \quad \rho \rightarrow \alpha \rho, \quad w \rightarrow \alpha w, \quad b \rightarrow \alpha b, \quad \chi \rightarrow \chi, \\
& \Omega \rightarrow \alpha \Omega, \quad E \rightarrow \alpha^{2} E, \quad j \rightarrow \alpha^{2} j, \\
& m \rightarrow \alpha m, \quad \mathcal{C} \rightarrow \alpha^{2} \mathcal{C}, \quad q \rightarrow \alpha^{4} q .
\end{aligned}
$$

The Lagrangian and the action transform homogeneously $\mathcal{L} \rightarrow \lambda^{2} \mathcal{L}$ and $S \rightarrow \lambda^{2} S$. By choosing $\alpha=1 / m$ in (2.29) we can make $m=1$ and deal with the remaining quantities in units of (the appropriate powers of) $m$.

\section{$3 \quad$ Types of embeddings}

The equations of motion written in (2.17) are potentially singular when

$$
b_{0}=\frac{w_{0}^{2}+\rho_{c}^{2}}{\Omega},
$$

where $b_{0}=b\left(\rho=\rho_{c}\right)$ and $w_{0}=w\left(\rho=\rho_{c}\right)$. The point $\rho=\rho_{c}$ where the condition (3.1) holds will be referred to as the pseudohorizon. Indeed, we will show below that $\rho=\rho_{c}$ is the event horizon of the induced open string metric on the D5-brane. The IR behaviour of the bulk fields when approaching this pseudohorizon determines three types of embeddings (see figure 1). We can have black hole embeddings (which cross the horizon), Minkowski embeddings (which do not intersect the pseudohorizon and reach the point $\rho=0$ ) and critical embeddings (for which the brane reaches the $\rho_{c}$ at $\rho_{c}=0$ ). In order to avoid the singularity at $\rho=\rho_{c}$ the first derivatives of the functions $w(\rho), b(\rho)$ and $\chi(\rho)$ have to be adjusted properly for black hole and critical embeddings.

\subsection{Black hole embeddings}

Expanding the functions $w(\rho), b(\rho)$ and $\chi(\rho)$ around $\rho=\rho_{c}$ as

$$
\begin{aligned}
w(\rho) & =w_{0}+w_{1}\left(\rho-\rho_{c}\right)+\cdots, \\
b(\rho) & =b_{0}+b_{1}\left(\rho-\rho_{c}\right)+\cdots, \\
\chi(\rho) & =\chi_{0}+\chi_{1}\left(\rho-\rho_{c}\right)+\cdots,
\end{aligned}
$$




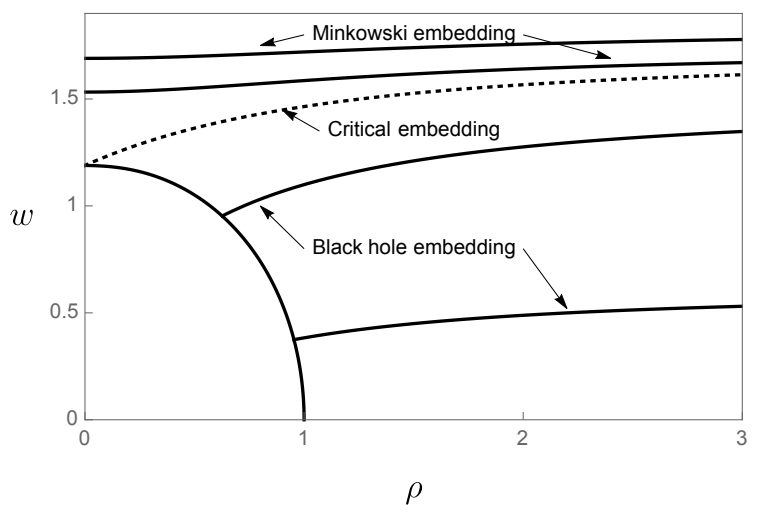

Figure 1. Profiles of the different types of embeddings for $E=\Omega=1$.

we obtain coefficients $w_{1}, b_{1}$ and $\chi_{1}$ whose explicit expressions are given in appendix A. Notice that $\chi_{1}$ appears in the expression of the on-shell lagrangian density $\mathcal{L}_{0}$ evaluated at the pseudohorizon $\rho=\rho_{c}$

$$
\mathcal{L}_{0}\left(\rho_{c}\right)=\rho_{c}^{2} b_{0} \chi_{1}
$$

We can use this expression to obtain the Joule heating $q$ in (2.16). Indeed as $q$ is constant, we can evaluate it at the pseudohorizon, with the result

$$
q=\Omega \rho_{c}^{2} b_{0}
$$

which only depends on $b_{0}$. Using (3.1), we get

$$
q=\rho_{c}^{2}\left(w_{0}^{2}+\rho_{c}^{2}\right) .
$$

\subsection{Minkowski embeddings}

In this case there is no $\rho_{c}$ that solves (3.1) and the brane reaches the origin $\rho=0$. We can therefore evaluate the Joule heating constant $q$ by taking $\rho=0$ in (2.18) and, since the right-hand side of this equation contains a $\rho^{4}$ factor we get $q=0$. Thus, for these embeddings there is no net energy flux on the boundary theory. Moreover, from (2.18) one readily concludes that the phase function $\chi(\rho)$ is in fact a constant. Expanding $b(\rho)$ and $w(\rho)$ around $\rho=0$

$$
\begin{gathered}
b(\rho)=b_{0}-\frac{b_{0} \Omega^{2}}{6\left(w_{0}^{4}-\Omega^{2} b_{0}^{2}\right)} \rho^{2}+\cdots, \\
w(\rho)=w_{0}+\frac{b_{0}^{2} \Omega^{2}}{3 w_{0}\left(w_{0}^{4}-\Omega^{2} b_{0}^{2}\right)} \rho^{2}+\cdots .
\end{gathered}
$$

\subsection{Critical embeddings}

When the effective horizon reaches the origin, $\rho_{c}=0$, the embedding is critical. The value of the $b$ field at the horizon is $b_{0}=w_{0}^{2} / \Omega, \chi(\rho)$ is also here constant and we can expand 
$b(\rho)$ and $w(\rho)$ around $\rho=0$, with the result

$$
\begin{aligned}
& b(\rho)=\frac{w_{0}^{2}}{\Omega}-\frac{\Omega}{2 \sqrt{\Omega^{2}+4 w_{0}^{2}}} \rho+\cdots \\
& w(\rho)=w_{0}+\frac{w_{0}}{\sqrt{\Omega^{2}+4 w_{0}^{2}}} \rho+\cdots .
\end{aligned}
$$

\subsection{Effective metric}

The effective open string metric $\gamma_{a b}$ is defined as [40]

$$
\gamma_{a b}=h_{a b}+\left(2 \pi \alpha^{\prime}\right) F_{a c} F_{b d} h^{c d},
$$

where we now denote by $h_{a b}$ the induced metric (2.5). Explicitly, for our ansatz, we have

$$
\begin{aligned}
\gamma_{a b} d \xi^{a} d \xi^{b}= & -F(\rho) d t^{2}+\frac{1+w^{\prime 2}+\left|c^{\prime}\right|^{2}}{\rho^{2}+w^{2}} d \rho^{2}-\frac{2 \Omega \operatorname{Im}\left(c c^{\prime *}\right)}{\rho^{2}+w^{2}} d t d \rho+F(\rho) e_{+} e_{-}+ \\
& +\frac{\rho^{2}+w^{2}}{4\left(1+w^{\prime 2}\right)}\left(e_{-} c^{\prime}+e_{+} c^{\prime *}\right)^{2}+\frac{\Omega^{2}}{4\left(\rho^{2}+w^{2}\right)}\left(e_{-} c+e_{+} c^{*}\right)^{2}+\frac{\rho^{2}}{\rho^{2}+w^{2}} d \Omega_{2}^{2},
\end{aligned}
$$

with $F(\rho)$ given by the following function

$$
F(\rho) \equiv \frac{\left(\rho^{2}+w^{2}\right)^{2}-\Omega^{2}|c|^{2}}{\rho^{2}+w^{2}},
$$

and $e_{ \pm}$are complex 1-forms

$$
e_{ \pm}=e^{\mp i \Omega t}(d x \pm i d y)
$$

The $(t, \rho)$ part of the metric gets diagonalized by means of the following change of coordinates

$$
d \tau=d t-A(\rho) d \rho, \quad d \rho_{*}=B(\rho) d \rho,
$$

where $A(\rho)$ and $B(\rho)$ are the following functions:

$$
A(\rho)=-\frac{\Omega \operatorname{Im}\left(c c^{*}\right)}{\left(\rho^{2}+w^{2}\right)^{2}-\Omega^{2}|c|^{2}}, \quad B(\rho)=\frac{\left(\rho^{2}+w^{2}\right) \mathcal{L}_{0}}{\rho^{2}\left[\left(\rho^{2}+w^{2}\right)^{2}-\Omega^{2}|c|^{2}\right]} .
$$

The new coordinate $\rho_{*}$ is just the Eddington-Finkelstein tortoise coordinate. In these coordinates, the transformations in (2.29) act as a rescaling $\left(\tau, \rho_{*}\right) \rightarrow \lambda^{-1}\left(\tau, \rho_{*}\right)$, and the effective metric takes the form

$$
\begin{aligned}
\gamma_{a b} d \xi^{a} d \xi^{b}= & -F(\rho)\left(-d \tau^{2}+d \rho_{*}^{2}\right)+F(\rho) e_{+} e_{-}+\frac{\rho^{2}+w^{2}}{4\left(1+w^{\prime 2}\right)}\left(e_{-} c^{\prime}+e_{+} c^{\prime *}\right)^{2}+ \\
& +\frac{\Omega^{2}}{4\left(\rho^{2}+w^{2}\right)}\left(e_{-} c+e_{+} c^{*}\right)^{2}+\frac{\rho^{2}}{\rho^{2}+w^{2}} d \Omega_{2}^{2} .
\end{aligned}
$$

The function $F(\rho)$ vanishes at $\rho=\rho_{c}$ and, therefore, this function acts as a blackening factor for the effective metric (3.14), with $\rho=\rho_{c}$ playing the role on an event horizon. The effective Hawking temperature $T_{H}$ is given by

$$
T_{H}=\frac{\kappa}{2 \pi}=-\left.\frac{\gamma_{t t}^{\prime}}{4 \pi \gamma_{t \rho}}\right|_{\rho=\rho_{c}} .
$$



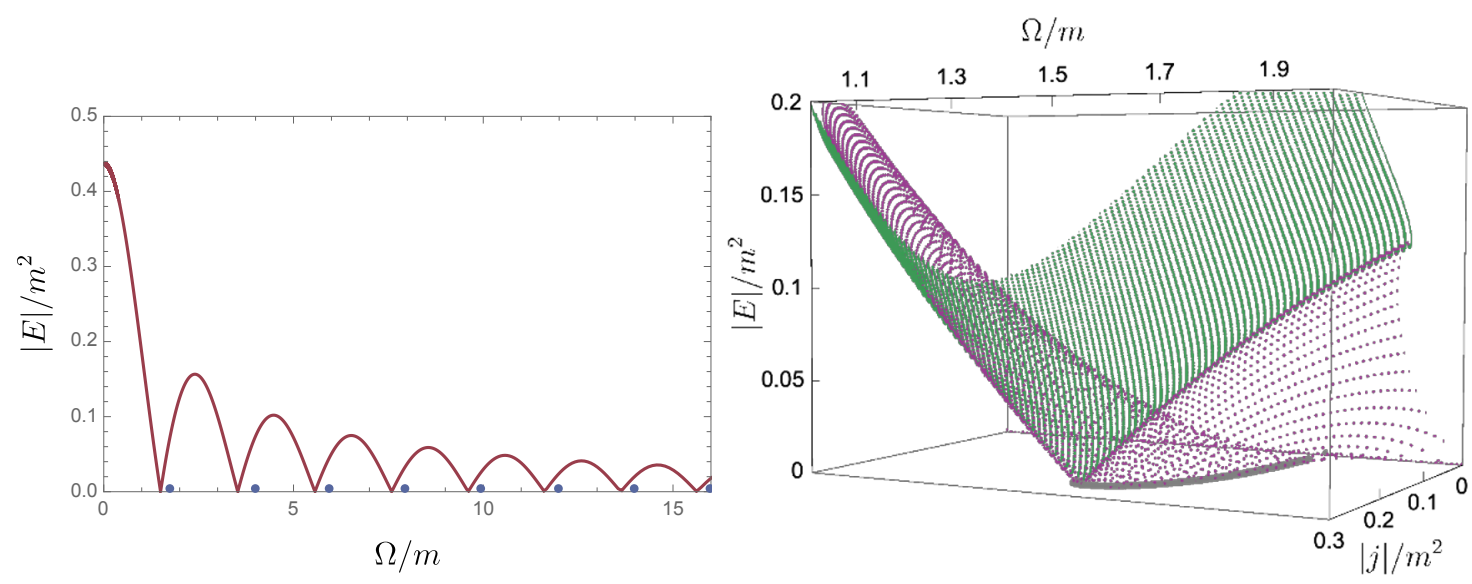

Figure 2. Electric field of the critical embeddings versus driving frequency. The frequencies for which $E$ vanish are the critical frequencies $\Omega_{c}$ of the vector meson Floquet condensates. The blue dots signal the frequencies (4.1) of the vector meson spectrum for different values of the principal quantum number $n$. On the right hand side, the three dimensional version of the left plot, focussing close to the first critical point, which stretches into a segment $\Omega / m \in(1.496,1.732)$.

Using the values of $\gamma_{t t}$ and $\gamma_{t \rho}$ found above, we get the $T_{H}$ in terms of near horizon data

$$
T_{H}=\left.\frac{2\left(\rho_{c}+w w^{\prime}\right)-\Omega b^{\prime}}{2 \pi \chi^{\prime} b}\right|_{\rho=\rho_{c}}=\frac{2\left(\rho_{c}+w_{0} w_{1}\right)-\Omega b_{1}}{2 \pi \chi_{1} b_{0}} .
$$

\section{Phase diagram}

In the previous section we have addressed the different types of embedding according to the IR boundary conditions. On the other hand, the phase diagram is constructed out of the UV boundary data that contain information about the sources and the responses. The analysis of the generic case proceeds by numerically integrating the system of equations (2.17). In this section we will obtain the precise boundary of the phase space of solutions presented in figures 2 and 3. Although the qualitative results are the same as the ones found in [27], we need the precise values of these boundaries in order to locate interesting places where to look for conductivity properties in the next section. As usual, integration proceeds from the IR to the UV. Indeed, enforcing regularity conditions found in section 3 as initial conditions at the pseudohorizon $\rho=\rho_{c}$ and integrating out up to the boundary yields a unique solution. For a given frequency $\Omega$ we can specify $b_{0}=b\left(\rho=\rho_{c}\right)$ and $w_{0}=w\left(\rho=\rho_{c}\right)$ and use the values of $w_{1}, b_{1}$ and $\chi_{1}$ computed in appendix $\mathrm{A}$ (for Minkowski embeddings $\rho_{c}=0$ and $\chi(\rho)$ is constant). From the UV behavior of the solutions $b(\rho), \chi(\rho)$ and $w(\rho)$ when $\rho \rightarrow \infty$ one can extract $E, j, m$ and $\mathcal{C}$. Due to the scaling symmetry (2.29) these quantities are not independent. It is natural to measure all quantities in terms of the quark mass $m$. Accordingly, we will present our results for $\Omega / m,|E| / m^{2}, j / m^{2}$ and $\mathcal{C} / m^{2}$.

In figure 2 we show the phase diagram in the parameter space $\left(\Omega / m,|E| / m^{2}\right)$. The solid curve is the locus of critical embeddings. We can think of it roughly as a boundary between the conductive phase (above) made of black hole embeddings, and insulator phase (below) 


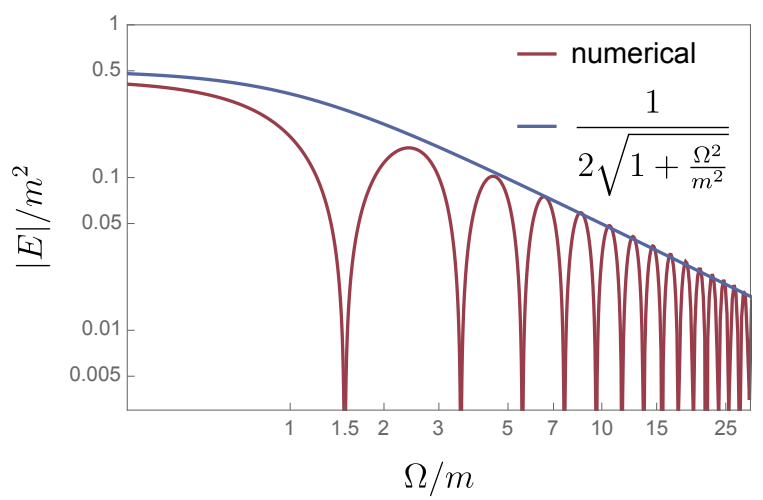

Figure 3. Logarithmic version of the plot in figure 2. The high $\Omega / m$ fitting is derived in section C.3 (see eq. (C.43)).
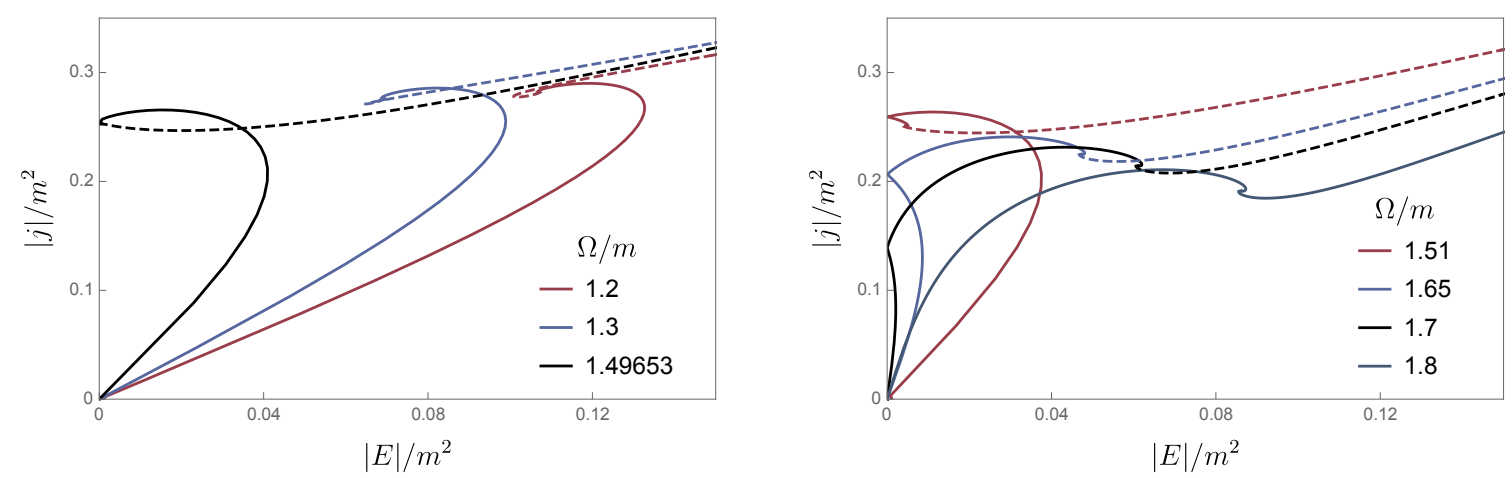

Figure 4. Electric current versus electric field for $\Omega \leq \Omega_{c}$ (left) and $\Omega>\Omega_{c}$ (right) around the first vector meson resonance. The solid curves correspond to the insulator (Minkowski) phase, whereas the dotted curves represent the conductive (black hole) phase. On the right curve we show that $E$ can only vanish for $j \neq 0$ for Minkowski embeddings with $\Omega_{c}=1.4965 \leq \Omega \leq 1.732=\Omega_{\text {meson }}$.

made of Minkowski embeddings. ${ }^{2}$ In figure 4 we plot the electric current versus the electric field for driving frequencies around the firs resonance. In figure 5 we represent the quark condensate for Minkowski and black hole embeddings and different driving frequencies. We notice in figures 2 and 3 that $|E| / m^{2}$ has a series of maxima whose values decrease as $\Omega / m$ increases. Moreover, for some discrete values $\Omega_{c} / m$ of the frequency the electric field vanishes and, as shown in figure 4 , the corresponding rotating current is non-zero.

Thus, for these frequencies we have a vector meson Floquet condensate of the type mentioned in the introduction. Actually, these $E=0$ Floquet states exist also for Minkowski embeddings in a finite range of frequency $\Omega_{c}<\Omega \leq \Omega_{\text {meson }}$, where $\Omega_{\text {meson }}$ is the mass of a vector meson in the defect theory. This mass spectra was computed in [32] and is given

\footnotetext{
${ }^{2}$ Although it is pretty clear from figure 1 that the critical embeddings lie in between Minkowski and Black Hole embeddings, the spiralling multivaluedness in the vicinity of the solid line (see figure 4 below) implies that one can find Black Hole embeddings closely below it, and also Minkowski embedding above.
} 

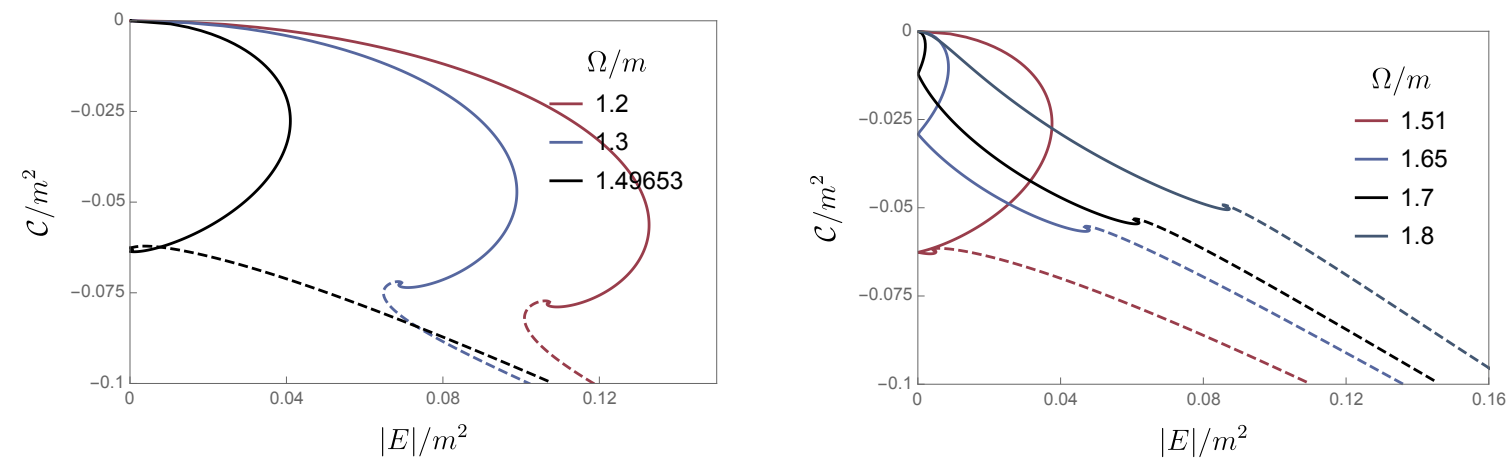

Figure 5. Quark condensate as a function of the electric field for several $\Omega$ around the first resonance. The curves on the left (right) are for $\Omega \leq \Omega_{c}\left(\Omega>\Omega_{c}\right)$. Solid (dashed) curves correspond to Minkowski (black hole) embeddings.
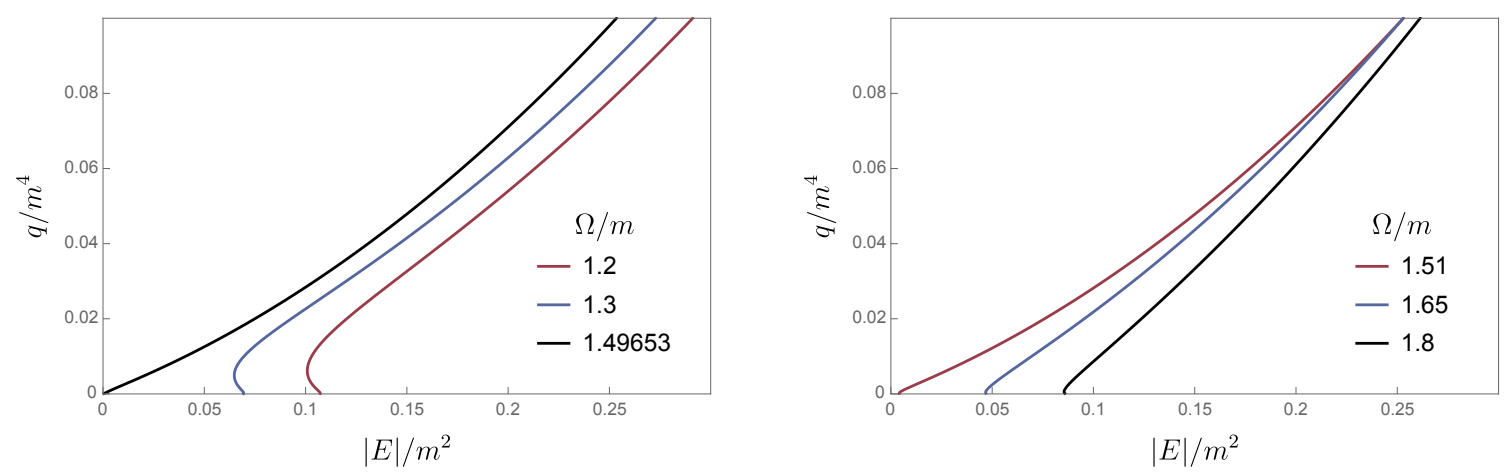

Figure 6. The Joule heating $q$ as a function of the electric field $E$ for black hole embeddings and several frequencies around the first resonance. On the left (right) plots $\Omega \leq \Omega_{c}\left(\Omega>\Omega_{c}\right)$.

by a tower of values depending on a principal quantum number $n$

$$
\Omega_{\text {meson }} / m=2 \sqrt{\left(n+\frac{1}{2}\right)\left(n+\frac{3}{2}\right)}, \quad n=0,1,2, \cdots .
$$

In section 5.2 and appendix $\mathrm{C}$ we obtain these frequencies by means of a linear analysis of the equations of motion. The first four values of the critical resonant frequencies and of the meson masses are

$$
\begin{aligned}
\Omega_{c} / m & =1.4965,3.5308,5.5676,7.5851 \cdots \\
\Omega_{\text {meson }} / m & =1.7320,3.8730,5.9161,7.9372, \cdots .
\end{aligned}
$$

In figure 6 we plot the Joule heating $q$ as a function of the electric field, whereas in figure 7 the Joule heating for black hole embeddings is plotted versus the driving frequency for several values of the electric field.

To get further insight on the structure of the phase diagram of the model, it is interesting to analyze in detail the relation between the current $j$ and the electric field $E$. Since $j$ and $E$ are vector quantities with two components in the $x y$ plane, we expect to have a 


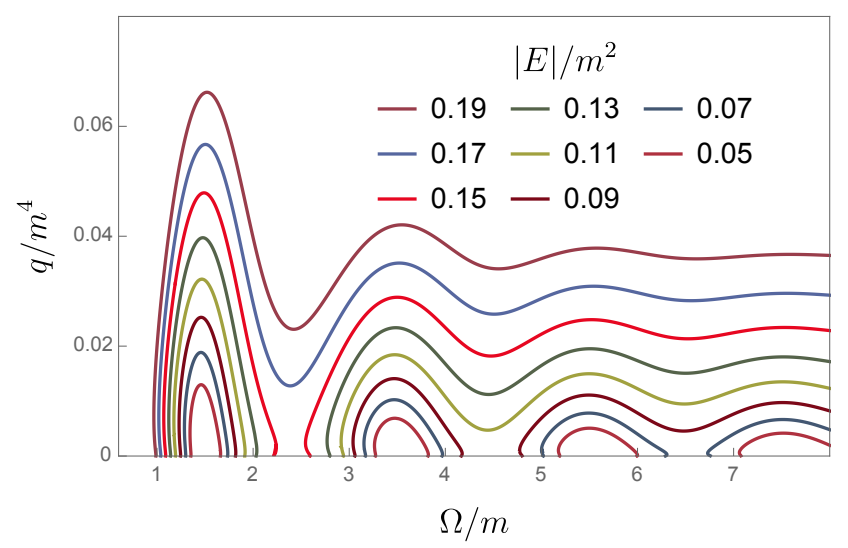

Figure 7. The Joule heating $q$ as a function of the frequency $\Omega$ for black hole embeddings and several values of the electric field.

relation of the type:

$$
\left(\begin{array}{c}
j_{x} \\
j_{y}
\end{array}\right)=\left(\begin{array}{cc}
\gamma_{x x} & \gamma_{x y} \\
-\gamma_{x y} & \gamma_{x x}
\end{array}\right)\left(\begin{array}{c}
E_{x} \\
E_{y}
\end{array}\right),
$$

where the form of the matrix is dictated by the rotational symmetry in the $x y$ plane. The matrix elements $\gamma_{x x}$ and $\gamma_{x y}$ are susceptibilities, defined in analogy with the longitudinal and transverse (Hall) conductivities. From the definitions (4.4) it is immediate to find $\gamma_{x x}$ and $\gamma_{x y}$ in terms of the components of $E$ and $j$ :

$$
\gamma_{x x}=\frac{E_{x} j_{x}+E_{y} j_{y}}{E_{x}^{2}+E_{y}^{2}}=\frac{|j|}{|E|} \cos \delta, \quad \gamma_{x y}=\frac{E_{y} j_{x}-E_{x} j_{y}}{E_{x}^{2}+E_{y}^{2}}=\frac{|j|}{|E|} \sin \delta,
$$

where $\delta$ is the angle formed by the vectors $\left(j_{x}, j_{y}\right)$ and $\left(E_{x}, E_{y}\right)$ in the $x y$ plane. The quantity $\gamma_{x x}$ is related to the Joule heating $q$. Indeed, one has $\gamma_{x x}=q /|E|^{2}$. Given this, and the fact that we have profusely illustrated the behaviour of $q$ in figures 6 and 7 , we shall focus on the study of $\gamma_{x y}$.

For massless black hole embeddings $\gamma_{x y}$ vanishes, since in this case $j$ and $E$ are colinear, as we demonstrate analytically in section 5.1 below. On the other hand $\gamma_{x y} \neq 0$ for Minkowski embeddings since there is no Joule heating in this case and the vectors $j$ and $E$ are orthogonal. This behavior can be verified by studying $\gamma_{x y}$ for black hole embeddings as a function of $\rho_{c}$ (see figure 8). When $\rho_{c}$ is large enough $\gamma_{x y} \rightarrow 0$, whereas it reaches a non-vanishing value as $\rho_{c} \rightarrow 0$. Interestingly, the sign of $\gamma_{x y}\left(\rho_{c} \rightarrow 0\right)$ changes at a discrete set of values of the driving frequency. We have identified these values as the critical resonant frequencies $\Omega_{c}$ of (4.3), as well as those for which the current $j$ vanishes for critical embeddings. These last frequencies with $j=0$ are very close to the maxima of the lobes in the $|E| / m^{2}$ versus $\Omega / m$ plot of figure 2 . The first numerical values of the frequencies which make $E$ maximal and $j$ zero for critical embeddings are:

$$
\begin{aligned}
\frac{\Omega}{m}(j=0) & =2.5080,4.5489,6.5769,8.5960, \cdots, \\
\frac{\Omega}{m}(E \text { maximal }) & =2.4008,4.4772,6.5270,8.5638, \cdots
\end{aligned}
$$



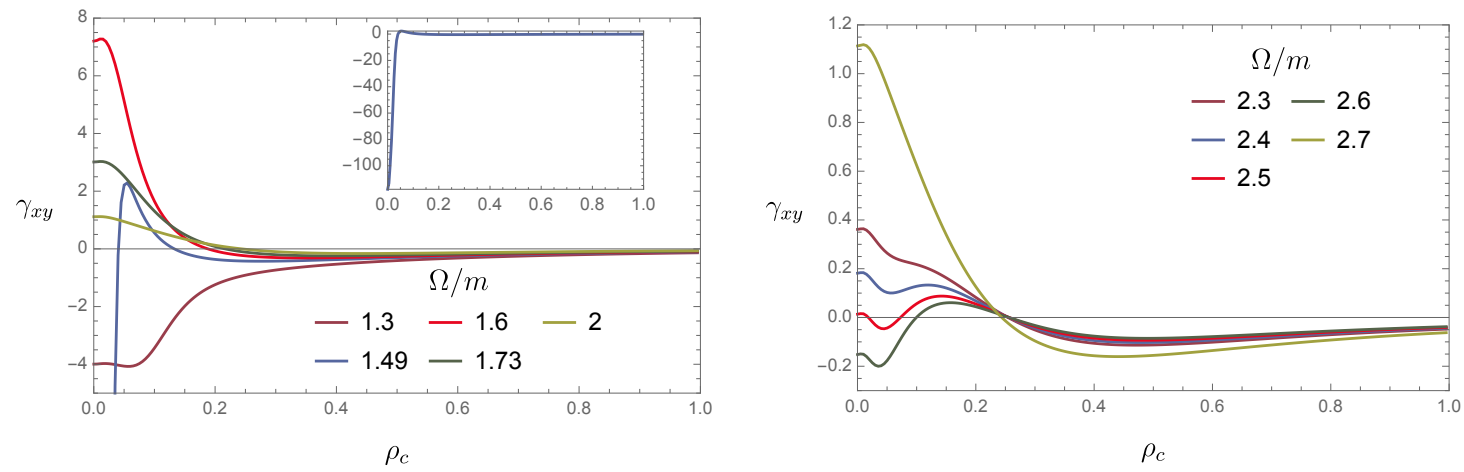

Figure 8. The susceptibility coefficient $\gamma_{x y}$ as a function of $\rho_{c}$ for black hole embeddings and different driving frequencies. On the left we plot the values for frequencies close to the first critical resonant frequency $\Omega_{c} / m=1.49$. On the right we represent $\gamma_{x y}$ for driving frequencies around $\Omega / m=2.5$, which is frequency of the first zero of $j$ for critical embeddings.
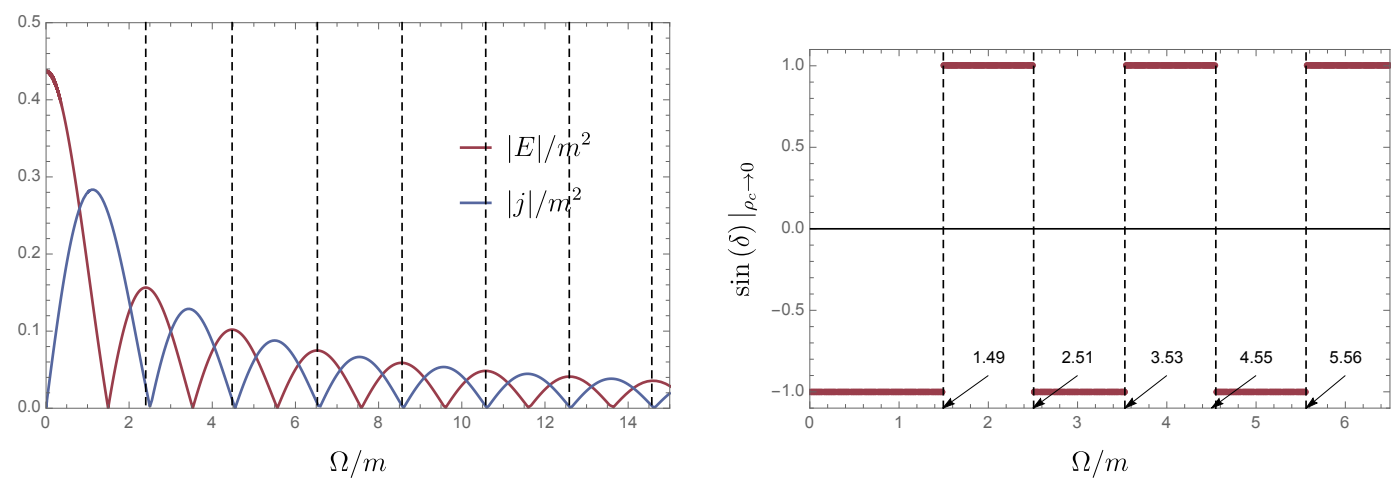

Figure 9. On the left we plot $|E| / \mathrm{m}^{2}$ and $j / \mathrm{m}^{2}$ versus the driving frequency for critical embeddings. On the right we depict the value of $\sin \delta$ for embeddings with $\rho_{c}=0$.

In figure 9 we plot together the $|E| / m^{2}$ and $j / m^{2}$ curves as a function of $\Omega / m$. We notice that the current curve also displays a lobe-shaped structure, displaced with respect to the $|E| / m^{2}$ curve, whose maxima (zeros) are very close to the zeros (maxima) of the $|E| / m^{2}$ curve. We have also checked that $\sin \delta\left(\rho_{c} \rightarrow 0\right)= \pm 1$ and changes discontinuously its sign at the discrete set of frequencies described above (see figure 9 ).

\section{$5 \quad$ Analytic solutions}

There are two limits where the equations are amenable to analytic solution. These are the low mass limit $m \rightarrow 0$, and the high frequency limit $\Omega \rightarrow \infty$. In the first case all embeddings are of black hole type. In the second, the three types of embeddings can occur.

\subsection{Massless embeddings}

Let us consider the particular case of massless black hole embeddings, which correspond to putting the embedding function $w(\rho)=0$. By inspection one easily concludes that $w=0$ 
is a consistent truncation of the equations of motion in (2.17). In this massless case, the lagrangian density (2.15) takes the form

$$
\mathcal{L}_{0}=\sqrt{\left(\rho^{4}-\Omega^{2} b^{2}\right)\left(1+b^{\prime 2}\right)+\rho^{4} b^{2}\left(\chi^{\prime}\right)^{2}} .
$$

As in the general case, $\chi(\rho)$ is a cyclic variable which, according to (2.18), is now given by

$$
\chi^{\prime}=\frac{q}{\rho^{2} b} \frac{\sqrt{\left(\rho^{4}-\Omega^{2} b^{2}\right)\left(1+b^{\prime 2}\right)}}{\sqrt{\Omega^{2} \rho^{4} b^{2}-q^{2}}} .
$$

Let us now eliminate $\chi(\rho)$ by introducing the Routhian $\mathcal{R}_{0}$, defined as

$$
\mathcal{R}_{0}=\chi^{\prime} \frac{\partial \mathcal{L}_{0}}{\partial \chi^{\prime}}-\mathcal{L}_{0}
$$

After some calculation, $\mathcal{R}_{0}$ as a function of $b(\rho)$ and $q$ becomes

$$
\mathcal{R}_{0}=-\sqrt{\left(1-\frac{q^{2}}{\Omega^{2} \rho^{4} b^{2}}\right)\left(\rho^{4}-\Omega^{2} b^{2}\right)} \sqrt{1+b^{\prime 2}} .
$$

As usual in this flavour brane setup, demanding reality of the Routhian fixes transport coefficients in terms of horizon data [50]. Indeed, at $\rho_{c}$ the two factors under the square root must change sign, and therefore vanish, simultaneously. This enforces $b_{0}=b\left(\rho_{c}\right)$ and $q$ to satisfy both equations (3.1) with $w_{0}=0$, and (3.4). Combined together, these equation entail, in the massless case

$$
q=\Omega^{2} b_{0}^{2}
$$

Let us look for solutions to the Euler Lagrange equations in which $b(\rho)$ is constant. Writing these equations in the following form

$$
\frac{\partial}{\partial b}\left[\left(1-\frac{q^{2}}{\Omega^{2} \rho^{4} b^{2}}\right)\left(\rho^{4}-\Omega^{2} b^{2}\right)\right]=-\frac{2}{b}\left(\Omega^{2} b^{2}-\frac{q^{2}}{\Omega^{2} b^{2}}\right),
$$

it's clear that there is a chance to have $b(\rho)$ constant precisely if it is equal to $b_{0}$ related to $q$ as in (5.5). Plugging this value of $b=b_{0}$ into the right-hand side of (5.2) we find

$$
\chi^{\prime 2}=\frac{\Omega^{2}}{\rho^{4}}
$$

that can be integrated right away

$$
\chi=-\frac{\Omega}{\rho}+\chi_{0},
$$

with $\chi_{0}$ constant. The minus sign is consistent with a positive Joule heating $q$, see (2.16), and infalling boundary conditions, see (2.28). Then $c(\rho)=b e^{i \chi}$ ends up being

$$
c(\rho)=\frac{\rho_{c}^{2}}{\Omega} e^{i \chi_{0}} e^{-i \frac{\Omega}{\rho}} .
$$


The extreme simplicity of this solution must be contrasted with the need for numerical integration in the D3-D7 setup [26]. By expanding near the UV as in (2.22), the electric field and current at the boundary are equal and given by

$$
E=j=-i \rho_{c}^{2} e^{i \chi_{0}}
$$

Hence, in the massless case, the rotating electric field and the induced current are aligned. The effective Hawking temperature for this massless case can be obtained from (3.16)

$$
T_{H}=\frac{\rho_{c}}{\pi b_{0} \chi^{\prime}\left(\rho_{c}\right)} .
$$

Using (5.7) and (3.1) this simplifies into

$$
T_{H}=\frac{\rho_{c}}{\pi}=\frac{q^{\frac{1}{4}}}{\pi} .
$$

Black hole embeddings with a small non-vanishing mass can be studied as perturbations of the exact massless solutions of this section. This analysis is carried out in detail in appendix B.

\subsection{High frequency limit}

If the frequency $\Omega$ is large, the boundary condition at the UV (2.22) fixes that $\mid c(\rho \rightarrow$ $\infty) \mid=E / \Omega \ll 1$ and this enforces $c(\rho)$ to be small for all values of $\rho$. The gauge field can then be treated as a perturbation around the state with $c=0$ and, as we shall see, admits an analytical solution. To start with, using the scaling symmetry (2.29) we can choose to linearize the equations of motion derived from (2.20) near the solution with $c=0$ and $w=1$. Writing $w=1+\delta w$ we get

$$
\begin{aligned}
c^{\prime \prime}+\frac{2}{\rho} c^{\prime}+\frac{\Omega^{2}}{\left(1+\rho^{2}\right)^{2}} c & =0, \\
\delta w^{\prime \prime}+\frac{2}{\rho} \delta w^{\prime} & =\frac{2 \Omega^{2}}{\left(1+\rho^{2}\right)^{3}}|c|^{2} .
\end{aligned}
$$

where $c$ and $\delta w$ are small. Notice that, although at linear order the equations decouple, as in [27] we have included the lowest order contribution to the coupling of modes, $\mathcal{O}\left(|\delta c|^{2}\right)$, in the equation of $\delta w$. The general solution of the equation of $\delta c$ is

$$
c(\rho)=c_{1} g_{1}(\rho)+c_{2} g_{2}(\rho),
$$

where $c_{1}$ and $c_{2}$ are constants and $g_{1}(\rho)$ and $g_{2}(\rho)$ are the functions

$$
\begin{aligned}
& g_{1}(\rho)=\frac{\sqrt{1+\rho^{2}}}{\rho} \sin \left(\sqrt{1+\Omega^{2}} \arctan (\rho)\right), \\
& g_{2}(\rho)=\frac{\sqrt{1+\rho^{2}}}{\rho} \cos \left(\sqrt{1+\Omega^{2}} \arctan (\rho)\right) .
\end{aligned}
$$


with asymptotic behaviour as $\rho \rightarrow \infty$ given by

$$
\begin{aligned}
& g_{1}(\rho)=\sin \left(\frac{\pi}{2} \sqrt{1+\Omega^{2}}\right)-\sqrt{1+\Omega^{2}} \cos \left(\frac{\pi}{2} \sqrt{1+\Omega^{2}}\right) \frac{1}{\rho}+\cdots, \\
& g_{2}(\rho)=\cos \left(\frac{\pi}{2} \sqrt{1+\Omega^{2}}\right)+\sqrt{1+\Omega^{2}} \sin \left(\frac{\pi}{2} \sqrt{1+\Omega^{2}}\right) \frac{1}{\rho}+\cdots .
\end{aligned}
$$

Similarly near $\rho=0, g_{1}$ and $g_{2}$ behave as

$$
\begin{aligned}
& g_{1}(\rho)=\sqrt{1+\Omega^{2}}-\frac{\Omega^{2} \sqrt{1+\Omega^{2}}}{6} \rho^{2}+\cdots \\
& g_{2}(\rho)=\frac{1}{\rho}-\frac{\Omega^{2}}{2} \rho+\cdots
\end{aligned}
$$

At the linearized level the Joule heating $q$ of eq. (2.26) is proportional to the Wronskian of $c$ and $c^{*}$

$$
q=i \frac{\Omega \rho^{2}}{2}\left(c c^{*}-c^{*} c^{\prime}\right)
$$

Since $q$ is independent of $\rho$, we could compute it by using the expansions (5.17) around $\rho=\infty$ or (5.18) around $\rho=0$. The result one obtains is the same and given by

$$
q=\Omega \sqrt{1+\Omega^{2}} \operatorname{Im}\left(c_{1} c_{2}^{*}\right) .
$$

The solution for $w(\rho)$ can be written as

$$
\delta w(\rho)=-\frac{2 \Omega^{2}}{\rho} \int_{0}^{\rho} \frac{s^{2}}{\left(s^{2}+1\right)^{3}}|c(s)|^{2}-2 \Omega^{2} \int_{\rho}^{\infty} \frac{s}{\left(s^{2}+1\right)^{3}}|c(s)|^{2}+c_{3},
$$

with $c_{3}$ being a new integration constant.

Since we obtained the general solution of our equations, we can now analyze the different types of embeddings. The details of this study are deferred to appendix C. Let us summarize here the results that we obtain there. (i) Regularity of the Minkowski embeddings at $\rho=0$ enforces that the constant $c_{2}$ in (5.15) has to vanish. (ii) By imposing the additional condition $E=0$ to these Minkowski solutions, we obtain the resonant frequencies (4.1), corresponding to the meson masses of the D3-D5 model. (iii) To construct black hole embeddings we have to build up a pseudohorizon in our linearized theory and impose the corresponding infalling boundary conditions. Although the linear approximation breaks down for the critical embeddings, it turns out that one can use the linear Minkowski solution to represent the critical configuration in the UV region of large $\rho$ and use this result to represent features of the phase diagram, such as the envelope curve of figure 3.

\section{Conductivities}

One of the main uses of the Floquet driving is the artificial engineering of materials exhibiting transport phenomena with topological character. In this sense, the obtention of a Hall conductivity without a magnetic field is an important hallmark. In the present context, this has been termed photovoltaic Hall effect [9] where it's origin has been shown 
to be topological, like the Thouless pumping. The time-reversal symmetry breaking and hence, the nontrivial band topology, is induced by the circularly polarized light. This proposal has been the subject of both theoretical and experimental studies during the last decade [55-59]. In [26] the photovoltaic Hall conductivity for massless carriers was obtained in the D3-D7 model, and speculated to behave consistently with the picture of a strongly coupled version of a $(3+1)$ Weyl semimetal. Our findings show similarities and departures from their results. For example, the massless case will be shown to be trivial. However, in the massive case, we will indeed find an intricate Hall effect.

Following the proposal in [10] we proceed to study the response of our system to a small additional electric field on the boundary pointing in a fixed direction. From this response we will be able to extract the $\mathrm{AC}$ and $\mathrm{DC}$ conductivities. We will closely mimic the strategy in [26] whose main steps will be reviewed and extended here for completeness, as our case involves three coupled field perturbations.

For the analysis it is most convenient to stick to the cartesian basis used in (2.6). On top of this background field, the idea is to place another fixed direction electric field

$$
\overrightarrow{\mathcal{E}}(t)=O(t) \vec{E}+\vec{\epsilon}(t),
$$

with $|\vec{\epsilon}| \ll|\vec{E}|$, and a harmonic (AC) time dependence

$$
\vec{\epsilon}(t)=\vec{\epsilon} e^{-i \omega t} .
$$

Our task is to extract the response of the system out of the non equilibrium steady state (NESS) solutions spelled out in the previous sections. In particular, the currents will also suffer a perturbation $\overrightarrow{\mathcal{J}}(t) \rightarrow O(t) \vec{j}+\delta \vec{j}(t)$ and one of our targets will be to extract the effective conductivity response matrix $\boldsymbol{\sigma}$, such that $\delta \vec{j}(t)=\boldsymbol{\sigma} \cdot \vec{\epsilon}(t)$.

From (6.1) we expect a perturbation of the bulk gauge field, which we also write now in cartesian components, $\vec{a}(t, \rho) \rightarrow \vec{a}(t, \rho)+\delta \vec{a}(t, \rho)$. The idea is now to refine the ansatz, taking into account the new driving data (6.1) and (6.2). First of all, let us reconsider the boundary conditions of the unperturbed gauge field, $\vec{a}(t, \rho)(2.7)$, now in cartesian coordinates. The near boundary expansion will be

$$
\begin{aligned}
\vec{a}(t, \rho) & =-\int^{t} \overrightarrow{\mathcal{E}}(t) d t+\frac{\overrightarrow{\mathcal{J}}}{\rho}+\ldots \\
& =-\frac{1}{\Omega} O(t) \varepsilon \vec{E}+\frac{\overrightarrow{\mathcal{J}}}{\rho}+\ldots
\end{aligned}
$$

where $\varepsilon$ stands for the $2 \times 2$ antisymmetric matrix

$$
\varepsilon=\left(\begin{array}{cc}
0 & 1 \\
-1 & 0
\end{array}\right) \text {. }
$$

The leading term matches the vector potential of the rotating electric field through $\overrightarrow{\mathcal{E}}(t)=$ $-\partial_{t} \vec{a}(t, \rho=\infty)$. This easily follows from the relation $\partial_{t} O \varepsilon=-\Omega O$. The boundary condition (6.3) suggests the following ansatz for the bulk solution

$$
\vec{a}(t, \rho)=O(t) \vec{c}(\rho)
$$


which is equivalent to (2.12) and (2.19) in cartesian basis. Near the boundary

$$
\vec{c}(\rho) \approx \vec{c}_{0}+\frac{\vec{c}_{1}}{\rho}+\ldots
$$

with $^{3}$

$$
\vec{c}_{0}=-\frac{1}{\Omega} \varepsilon \vec{E}, \quad \overrightarrow{\mathcal{J}}(t)=O(t) \vec{c}_{1}
$$

Considering the perturbations, now the bulk gauge potential $\vec{a}+\delta \vec{a}$ has to match the full electric field (6.1) at the boundary, $-\partial_{t}(\vec{a}+\delta a)=\overrightarrow{\mathcal{E}}(t)$

$$
\vec{a}(t, \rho=\infty)+\delta \vec{a}(t, \rho=\infty)=-\frac{1}{\Omega} O(t) \varepsilon \vec{E}-\frac{i}{\omega} \vec{\epsilon} e^{-i \omega t} .
$$

Induced by this new boundary conditions, the ansatz will assume that the static field, $c(\rho)$, develops a time dependent perturbation

$$
\vec{a}(t, \rho)+\delta \vec{a}(t, \rho)=O(t)(\vec{c}(\rho)+\delta \vec{c}(t, \rho)) .
$$

When the flavour branes are massive, these fluctuations couple to a perturbation of the brane embedding functions $w(\rho) \rightarrow w(\rho)+\delta w(t, \rho)$. In this case we will be dealing with a 3 component vector of fluctuations $\delta \vec{\xi}(t, \rho)=\left(\delta c_{x}, \delta c_{y}, \delta \omega\right)$. The perturbed equations of motion, linear in $\delta \vec{\xi}$, will assume the following form, in terms of the $\left(\tau, \rho_{*}\right)$ coordinates (see eq. (3.12))

$$
\left(\partial_{\tau}^{2}-\partial_{\rho_{*}}^{2}+\mathbf{A}(\rho) \partial_{\tau}+\mathbf{B}(\rho) \partial_{\rho_{*}}+\mathbf{C}(\rho)\right) \delta \vec{\xi}=0 .
$$

Here, $\mathbf{A}, \mathbf{B}$ and $\mathbf{C}$ are $3 \times 3$ matrices depending on the radial coordinate $\rho\left(\rho^{*}\right)$ and, parametrically, on the rotating frequency $\Omega$. At the pseudo-horizon $\rho=\rho_{c}\left(\rho^{*} \rightarrow-\infty\right)$ one finds

$$
\mathbf{A}\left(\rho=\rho_{c}\right)=-\mathbf{B}\left(\rho=\rho_{c}\right) \equiv \mathbf{A}_{c} \quad \mathbf{C}\left(\rho=\rho_{c}\right)=0 .
$$

Thus, in this limit, $\rho^{*} \rightarrow-\infty$, the fluctuation equations become

$$
\left(\partial_{\tau}^{2}-\partial_{\rho_{*}}^{2}\right) \delta \vec{\xi}+\mathbf{A}_{c}\left(\partial_{\tau}-\partial_{\rho_{*}}\right) \delta \vec{\xi}=0
$$

whose general solution takes the form

$$
\delta \vec{\xi}=\vec{f}\left(\tau+\rho_{*}\right)+e^{-\mathbf{A}_{c} \rho_{*}} \vec{g}\left(\tau-\rho_{*}\right),
$$

with $\vec{f}$ and $\vec{g}$ arbitrary vector functions. Imposing that $\vec{g}=0$ selects the ingoing wave boundary condition at the pseudohorizon.

Let us now work out the boundary conditions for the fluctuations. First, we want to keep the mass of the flavor brane fixed. After inserting (6.9) into (6.8) and multiplying by $\mathcal{O}^{-1}$, the boundary conditions for $\delta \vec{\xi}(t, \infty)$ turn out to be

$$
\delta \vec{c}(t, \rho=\infty)=-\frac{i}{\omega} O(-t) e^{-i \omega t} \vec{\epsilon}, \quad \delta \omega(t, \rho=\infty)=0 .
$$

\footnotetext{
${ }^{3}$ This replaces $(2.22)$ with $j=c_{1 x}+i c_{1 y}$.
} 
Following [26], we expand the matrix $\mathcal{O}(t)$, defined in (2.6) as

$$
O(t)=\mathbf{M}_{+} e^{i \Omega t}+\mathbf{M}_{-} e^{-i \Omega t},
$$

where $\mathbf{M}_{ \pm}$are orthogonal projectors given by

$$
\mathbf{M}_{ \pm}=\frac{1}{2}\left(\begin{array}{cc}
1 & \pm i \\
\mp i & 1
\end{array}\right)
$$

Finally, defininig $\omega_{ \pm}=\omega \pm \Omega$, the boundary UV condition (6.14) of $\delta \vec{c}$ can be written as

$$
\delta \vec{c}(t, \rho=\infty)=-\frac{i}{\omega}\left(\mathbf{M}_{+} e^{-i \omega_{+} t}+\mathbf{M}_{-} e^{-i \omega_{-} t}\right) \vec{\epsilon} .
$$

It is natural to assume that from these boundary condutions the bulk fluctuations $\delta \vec{c}(t, \rho)$ will oscillate with frequencies

$$
\delta \vec{c}(t, \rho)=\vec{\beta}_{+}(\rho) e^{-i \omega_{+} t}+\vec{\beta}_{-}(\rho) e^{-i \omega_{-} t} .
$$

As a consequence of the coupling in the linearized equations, we will assume likewise that the embedding fluctuations will resonate with the same frecuencies

$$
\delta w(t, \rho)=\gamma_{+}(\rho) e^{-i \omega_{+} t}+\gamma_{-}(\rho) e^{-i \omega_{-} t} .
$$

In summary, setting $\delta \vec{\xi}(t, \rho)=\vec{\xi}_{ \pm}(\rho) e^{-i \omega_{ \pm} t}$, the 3 component vectors $\vec{\xi}_{ \pm}(\rho)=\left(\beta_{ \pm, x}, \beta_{ \pm, y}, \gamma_{ \pm}\right)$ will satisfy the linearized ordinary differential system

$$
\left[\frac{d^{2}}{d \rho_{*}^{2}}-\mathbf{B}(\rho) \frac{d}{d \rho^{*}}+\omega_{ \pm}^{2}+i \omega_{ \pm} \mathbf{A}(\rho)-\mathbf{C}(\rho)\right] \vec{\xi}_{ \pm}=0
$$

From (6.14), the brane fluctuations have vanishing boundary source $\gamma_{ \pm}(\infty)=0$. Inserting the boundary expansions for the fields in (6.18)

$$
\delta \vec{c}(t, \rho) \approx \delta \vec{c}^{(0)}(t)+\frac{\delta \vec{c}^{(1)}(t)}{\rho}+\ldots, \quad \vec{\beta}_{ \pm}(\rho)=\vec{\beta}^{(0)}+\frac{\vec{\beta}^{(1)}}{\rho}+\ldots
$$

and comparing we get

$$
\delta \vec{c}^{(0)}(t)=\vec{\beta}_{+}^{(0)} e^{-i \omega_{+} t}+\vec{\beta}_{-}^{(0)} e^{-i \omega_{-} t}, \quad \delta \vec{c}^{(1)}(t)=\vec{\beta}_{+}^{(1)} e^{-i \omega_{+} t}+\vec{\beta}_{-}^{(1)} e^{-i \omega_{-} t} .
$$

Comparing the first of these equations with (6.17) we conclude that

$$
\vec{\beta}_{ \pm}^{(0)}=-\frac{i}{\omega} \mathbf{M}_{ \pm} \vec{\epsilon}
$$

The subleading vectors $\vec{\beta}_{ \pm}^{(1)}$ determine the variation of the current $\delta \overrightarrow{\mathcal{J}}(t)=O(t) \delta \vec{c}^{(1)}(t)$. Using (6.7), (6.15) and (6.17)

$$
\delta \overrightarrow{\mathcal{J}}(t)=e^{-i \omega t}\left(\mathbf{M}_{+} \vec{\beta}_{+}^{(1)}+\mathbf{M}_{-} \vec{\beta}_{-}^{(1)}+\mathbf{M}_{+} \vec{\beta}_{-}^{(1)} e^{2 i \Omega t}+\mathbf{M}_{-} \vec{\beta}_{+}^{(1)} e^{-2 i \Omega t}\right) .
$$


As usual in the AdS/CFT program, given a regular solution, the vectors $\vec{\beta}_{ \pm}^{(1)}$ and $\vec{\beta}_{ \pm}^{(0)}$ will no more be independent. From the linearity of the equations of motion it follows that this relation is also linear

$$
\vec{\beta}_{ \pm}^{(1)}=\mathbf{X}_{ \pm} \vec{\beta}_{ \pm}^{(0)}=-\frac{i}{\omega} \mathbf{X}_{ \pm} \mathbf{M}_{ \pm} \vec{\epsilon}
$$

where $\mathbf{X}_{ \pm}$are $2 \times 2$ matrices to be computed and (6.23) has been used. Plugging this into (6.24) allows to find a relation between the current $\delta \mathcal{J}$ and the applied electric field $\vec{\epsilon}$ which defines $\boldsymbol{\sigma}, \boldsymbol{\sigma}_{+}$and $\boldsymbol{\sigma}_{-}$as the $2 \times 2$ conductivity matrices of the three current modes, one with frequency $\omega$ and two with $\omega \pm 2 \Omega$ (the heterodyning mixing modes [53])

$$
\delta \overrightarrow{\mathcal{J}}=\left[\boldsymbol{\sigma}(\omega) e^{-i \omega t}+\boldsymbol{\sigma}^{+}(\omega) e^{-i(\omega+2 \Omega) t}+\boldsymbol{\sigma}^{-}(\omega) e^{-i(\omega-2 \Omega) t}\right] \vec{\epsilon},
$$

where

$$
\begin{aligned}
\boldsymbol{\sigma}(\omega) & =-\frac{i}{\omega}\left(\mathbf{M}_{+} \mathbf{X}_{+} \mathbf{M}_{+}+\mathbf{M}_{-} \mathbf{X}_{-} \mathbf{M}_{-}\right) \\
\boldsymbol{\sigma}^{+}(\omega) & =-\frac{i}{\omega} \mathbf{M}_{-} \mathbf{X}_{-} \mathbf{M}_{+} \\
\boldsymbol{\sigma}^{-}(\omega) & =-\frac{i}{\omega} \mathbf{M}_{+} \mathbf{X}_{+} \mathbf{M}_{-} .
\end{aligned}
$$

The procedure to compute $\mathbf{X}_{ \pm}$starts by solving the differential equation system (6.20) from the pseudohorizon $\rho_{*} \rightarrow-\infty$ to the AdS boundary at $\rho_{*}=0$. At the horizon we impose the ingoing boundary condition $\vec{\xi}_{ \pm}\left(\rho_{*}\right) \simeq \vec{c} e^{-i \omega_{ \pm} \rho_{*}}$, where $\vec{c}$ are constant vectors $(1,0,0),(0,1,0)$ and $(0,0,1)$. Due to linearity of the equations we obtain in this way two complex $3 \times 3$ matrices $P_{ \pm}$and $Q_{ \pm}$such that $\vec{\xi}_{ \pm}^{(0)}=P_{ \pm} \vec{c}_{ \pm}$, and $\vec{\xi}_{ \pm}^{(1)}=Q_{ \pm} \vec{c}_{ \pm}$. In this way we can solve for $\vec{\xi}_{ \pm}^{(1)}=Q_{ \pm} P_{ \pm}^{-1} \vec{\xi}_{ \pm}^{(0)}$. Since we will be setting the source for the embedding fluctuations to zero, i.e. $\xi_{ \pm, 3}^{(0)} \equiv \gamma_{ \pm}^{(0)}=0$ and we only care about $\xi_{ \pm, 1,2}^{(1)}$, the searched for matrices $\mathbf{X}_{ \pm}$in (6.25) will be given by the $2 \times 2$ submatrix of the product $Q_{ \pm} P_{ \pm}^{-1}$

$$
\mathbf{X}_{ \pm i j}=\left[Q_{ \pm} P_{ \pm}^{-1}\right]_{i j=1,2}
$$

From them, the conductivities are extracted using (6.27).

\subsection{AC conductivities}

The results for the photovoltaic optical Hall conductivity, $\sigma_{x y}$, as well as for the photovoltaic optical absortion spectrum, $\sigma_{x x}$, can be seen in figure 10. We have plotted everything in units of the carrier mass $m .{ }^{4}$ The driving laser has been set to the first resonant frequency $\Omega / m=1.496$ for a discrete set of values of $|E| / m^{2}$ (see the coloured dots along the vertical dashed line in figure 12).

The behaviour in all components of $\boldsymbol{\sigma}$ shows oscillations whose amplitude grows for decreasing $|E| / m^{2}$. All conductivities oscillate around 0 except for $\operatorname{Re}\left(\sigma_{x x}\right)$ which does it around a saturation value 1 .

\footnotetext{
${ }^{4}$ Numerically we have performed the integration for 2 different sets of values of $(E, \Omega, m)$ in order to verify the scaling symmetry of eq. (2.29).
} 


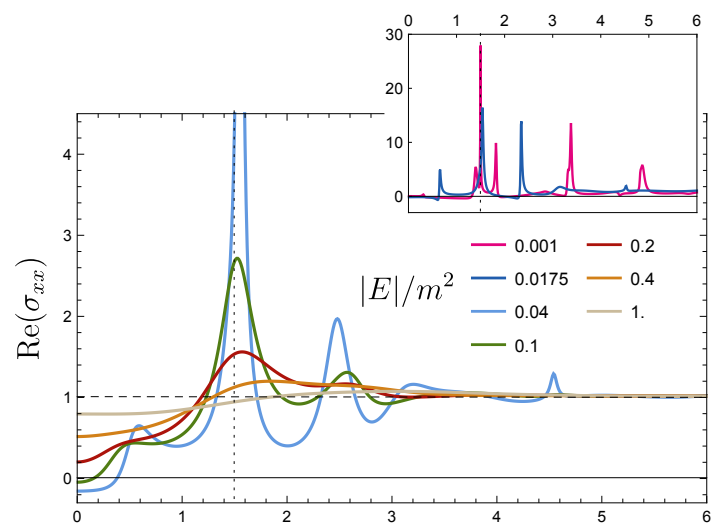

$\omega / m$

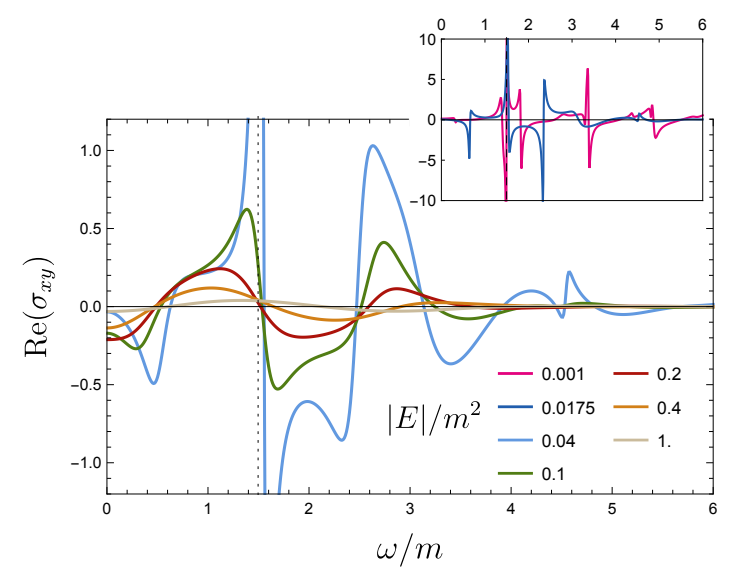

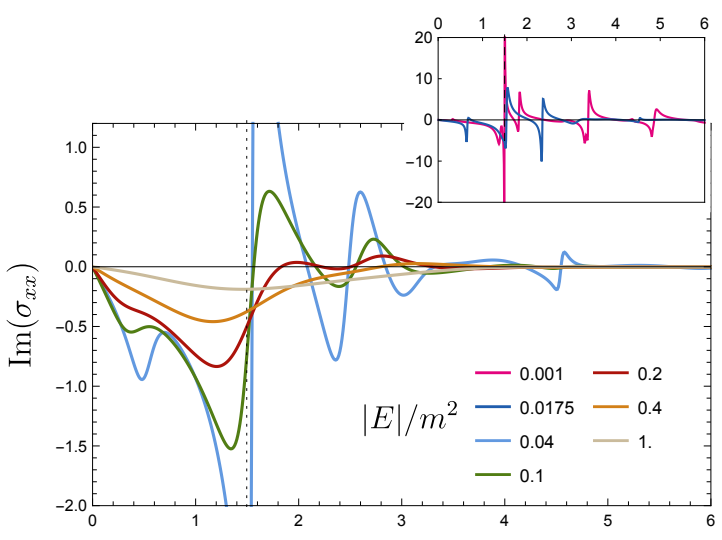

$\omega / m$

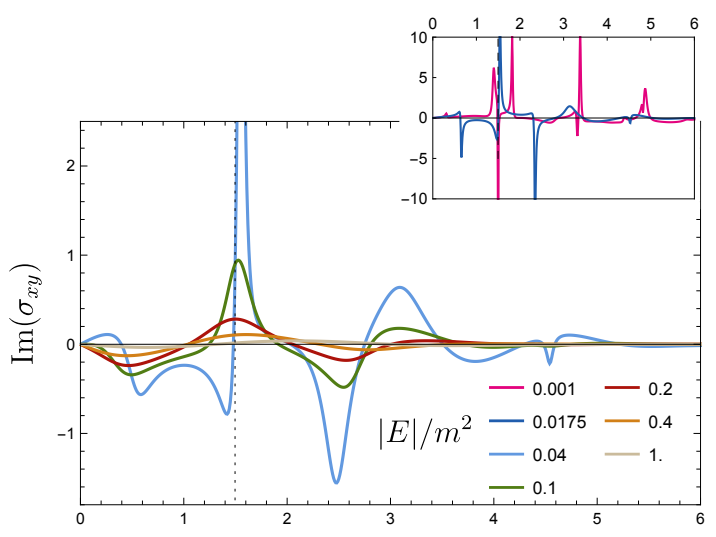

$\omega / m$

Figure 10. AC conductivities for fixed value of $\Omega / m=1.496$ and different values of $|E| / m^{2}$. They correspond to the vertically aligned dots in figure 12 with the same color code. In the insets, the curves at low values of $|E| / m^{2} \rightarrow 0$ deep into the critical wedge, where they develop peak resonances. The value $\omega / m=\Omega / m=1.496$ is signalled with a dashed vertical line.

The departure in $(2+1)$ dimensions, from the analog $(3+1)$ dimensional holographic system is significant (see figure 7 and 8 in [26]). In both cases, in the large frequency limit, the conductivity becomes proportional to the identity matrix $\boldsymbol{\sigma}(\omega \rightarrow \infty)=c \mathbb{I}$. In the D3/D7 case, $c$ is a complex linearly growing function of $\omega$, whereas in our case $c$ is a constant (equal to 1 in our normalization in this section, see later however). This asymptotic behaviour is also attained in the limit of high $|E| / m^{2} \gg 1$, as controlled by the massless limit $m \rightarrow 0$ which is analytically solvable. Not only the amplitude of the maxima, but also their movement is different. In the D3/D7 case their positions shift to lower frequency for decreasing external field. Here we see that they tend to approach the critical $\omega=1.496$.

As $|E| / m^{2}$ becomes smaller, strong oscillations develop, with the most prominent one sharpening around the resonance value for the optical and driving frequencies $\omega=\Omega$ (signalled with a dotted vertical line in all the plots). This feature bears resemblance with the one obtained in [10] (Fig 1b), although the behaviour of the peak amplitude with the intensity is the opposite. 
In the limit $|E| / m^{2} \rightarrow 0$ these oscillations become very sharp peaks as shown in the insets in figures 10. As we move down towards $E / m^{2} \rightarrow 0$ the shape and positions of these peaks change rapidly. This can be appreaciated in the insets, where the curves for $E / m^{2}=0.0175$ and 0.001 look decorrelated. This last magenta curve shows a subseries of peaks at frequencies $\omega=1.496,3.38,4.87, \ldots$ which have some overlap with the vector meson Floquet condensate spectrum $\omega=1.496,3.53,5.56, \ldots$ at shifted values.

The fact that $\boldsymbol{\sigma}$ is dimensionless in $(2+1)$ dimensions eases the comparison with results in the literature. For the Hall component, $\sigma_{x y}(\omega)$ there is a striking similarity with the plot in figures 8 of [12], where also the resonance peak is visible as well as secondary peaks. Importantly, in the limit $E \rightarrow 0$ the photoelectric Hall conductivity does not vanish. This signals spontaneous time reversal symmetry breaking and might be a consequence of the underlying vector meson Floquet condensate state. ${ }^{5}$

The enhancement of the oscillations and peak formation observed in figure 10 is a universal feature as we approach the line of critical embeddings. We have followed the movement of the peaks for a sequence of configurations very closely above the critical line, for background frequencies in the range $\Omega / m \in(1.3,2.32)$ (see figure 2). The resulting curves are plotted in figure 11. A remarkable fact is the presence of one peak whose position barely changes and is confined to the interval $\omega / m \in(1.4965,1.73)$, i.e. among the vector meson Floquet condensate frequency and the meson mass resonance (see figure 2 and eqs. (4.2), (4.3)). Very slightly above the critical line there are two sidewise peaks which appear within a short interval in $E / \mathrm{m}^{2}$. They all can be seen also in figure 11. The remarkable fact about these lateral peaks is that they shift position with the driving in such a way that their mean value coincides with the background frequency $\Omega$, while their splitting is almost constant $\Delta \omega / m \sim 1.7$. This curious result is very neatly seen on embeddings to the right of the vector meson Floquet condensate, i.e. in the segment $\Omega / m \in(1.496,2.32)$ and, very much damped, nearby to its left. The blue curve at $\Omega / m=1.496$ is the same one that can be observed in the insets of figure 10 , which has $E / m^{2}=0.0175$. At this frequency $\Omega=\Omega_{c}$, there are embedding solutions down to $E=0$ and this gives the magenta curves in those insets at values of $E / m^{2}=0.001$ and even lower.

We also computed the heterodyning conductivities $\boldsymbol{\sigma}^{ \pm}(\omega)$. They bear the same features as for the normal conductivity, and the reader can found further information in appendix E. Roughly stated, the curves for these exotic conductivities share, each of them, half of the peaks visible in $\boldsymbol{\sigma}(\omega)$.

\subsection{DC conductivities}

The zero frequency limit $\omega \rightarrow 0$ is formally singular (cf. (6.14)). However the numerical value of the $\mathrm{AC}$ conductivity is well behaved and stable down to values of $\omega / \Omega$ well below $10^{-3}$. Hence we will use this value to define our DC conductivity. The authors of [26] performed a numerical analysis in the time domain and showed consistent agreement between the two prescriptions. From the plots in figures 10, it is apparent that the imaginary

\footnotetext{
${ }^{5}$ We thank T. Oka for this observation.
} 

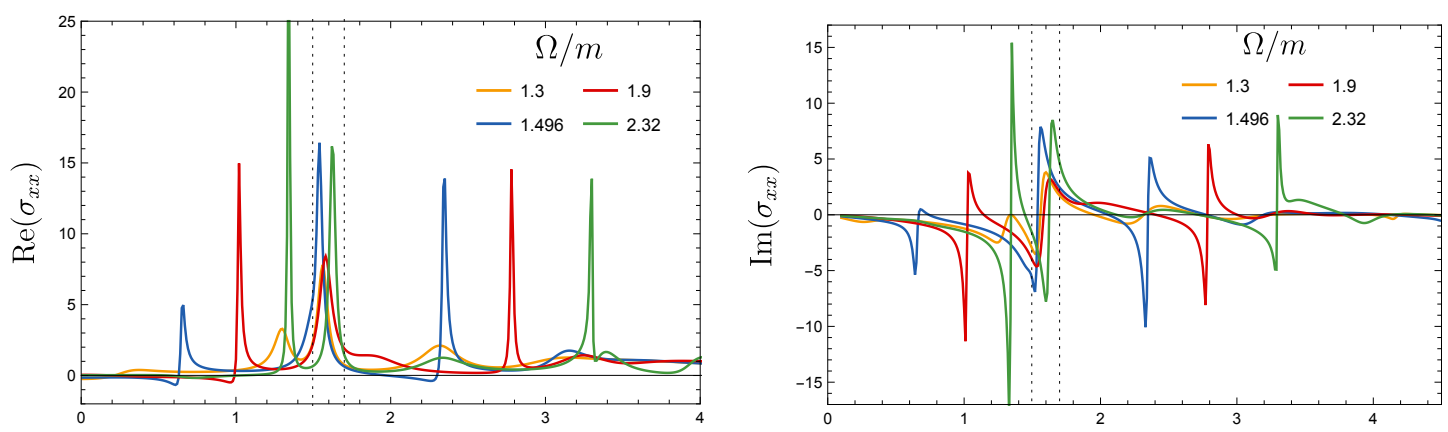

$\omega / m$

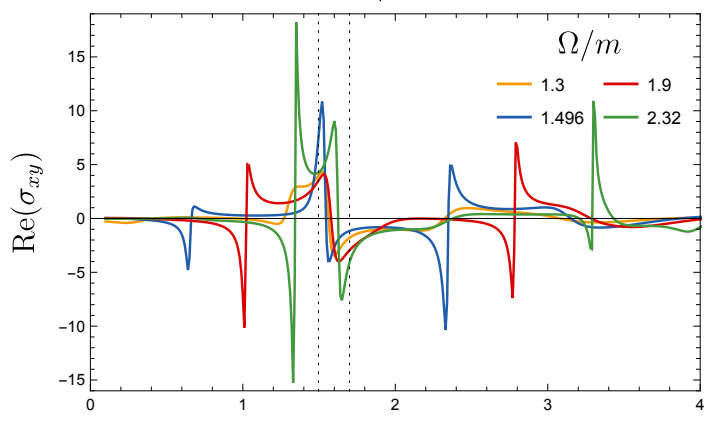

$\omega / m$

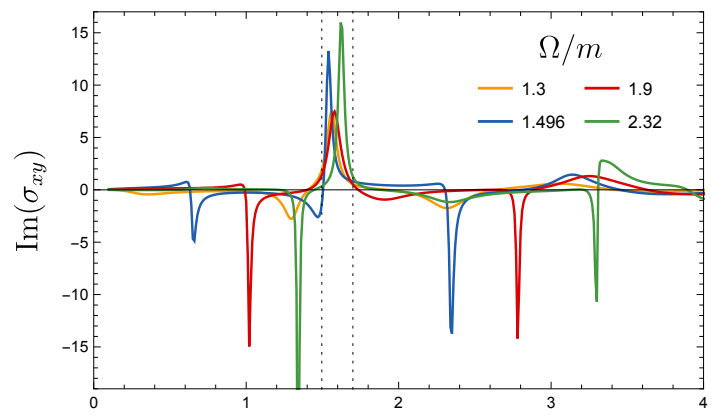

$\omega / m$

$\omega / m$

Figure 11. Peaks in the AC conductivities closely above along the critical line. The exact position of these curves in phase space can be seen in figure 12 as coloured circles. The vertical dashed lines signal the vector meson Floquet condensate and the meson mass frequencies respectively. The central peaks bunch in this region whereas the two lateral peaks move with $\omega$ while keeping their separation almost constant, $\Delta \omega / m \sim 1.7$.

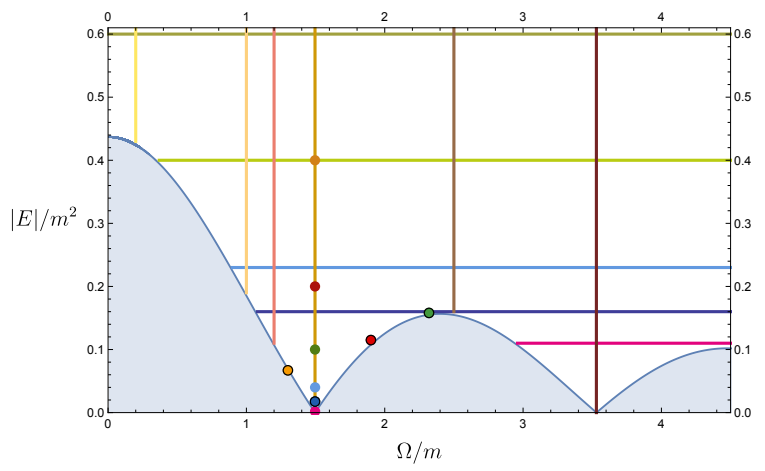

Figure 12. The dots and circles signal the points where the $\mathrm{AC}$ conductivities in figures 10 and 11 have been plotted respectively. Also shown are the lines with either $\Omega / m$ or $|E| / m^{2}$ fixed, used to plot the DC conductivities in figures 13 and 14. In all cases, the same color code has been used.

parts vanish in the limit $\omega \rightarrow 0$. Hence the DC conductivities are real, as follows from the relation $\boldsymbol{\sigma}(-\omega)=\boldsymbol{\sigma}(\omega)^{*}$.

Figures 13 and 14 summarize our numerical results. In figure 13 we exhibit $\boldsymbol{\sigma}(\omega=0)$ for varying $\Omega / m$ along lines of constant $|E| / m^{2}$. For high enough $E / m^{2}$ the limit $\Omega \rightarrow 0$ stays in the conducting phase and the direct conductivity $\sigma_{x x}$ has the correct static limit 

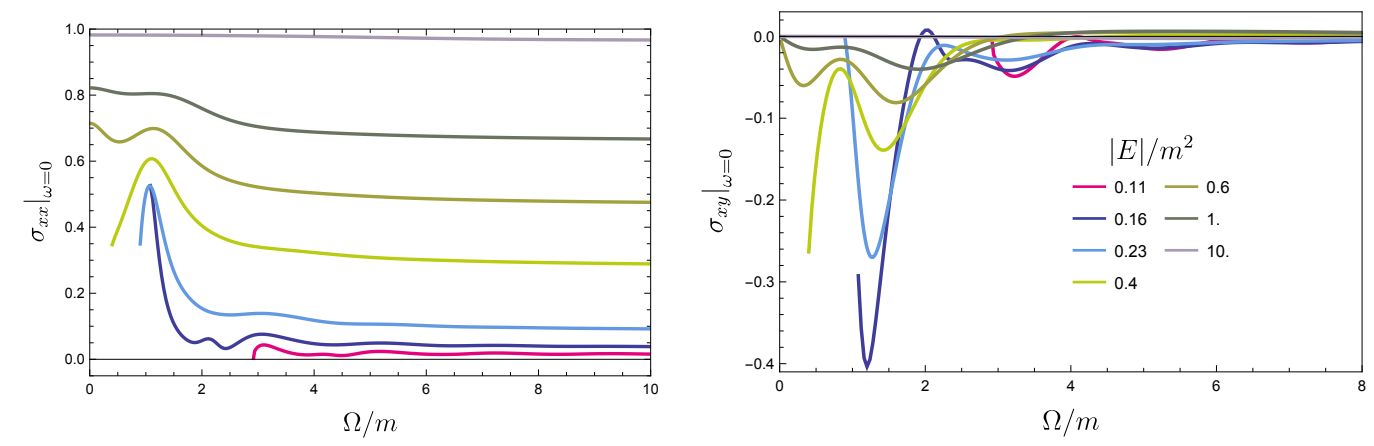

Figure 13. DC Conductivities as a function of $\Omega / m$ for various values of $|E| / m^{2}$.
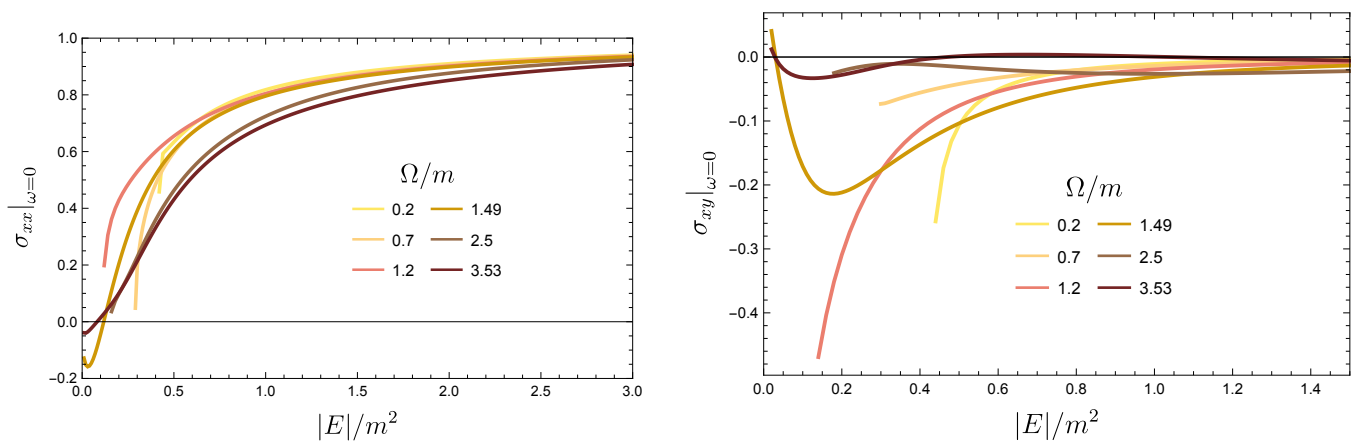

Figure 14. DC Conductivities as a function of $|E| / m^{2}$ for various values of $\Omega / m$.

obtained in [50] where it becomes a constant that only depends on $m$, and saturates to a maximum value equal to one (see section 6.3 below for the correct normalization).

In figure 14 we plot $\boldsymbol{\sigma}(\omega=0)$, varying $|E| / \mathrm{m}^{2}$ along lines of constant $\Omega / m$. Our results should be compared with the condensed matter calculation in [9] (see the discussion after figure2). For the Hall conductivity we see qualitative agreement in that, in absolute value, $\left|\sigma_{x y}\right|$ first increases with $|E| / m^{2}$ and then decreases. For high $\Omega / m(\sim 3.53)$ we find values where this conductivity has zeros, this being a remarkable feature. For $\sigma_{x x}$ we find a monotonic growth that reaches a limiting value for high $|E| / m^{2}$. In contrast, authors of [9] (see figure2b) obtained an intermediate valley which they proposed to be a reflection of the gap opening at the Dirac points. If so, our model is too crude to capture this feature.

The comparison with the $(3+1)$ case is complicated because the authors of [26] only considered the massless case, which in our setup turns out to be analytic and trivial. Indeed, taking $\left(|E| / m^{2}, \Omega / m\right) \rightarrow \infty$ the Hall conductivity vanishes whereas the DC conductivity asymptotes to a constant value. One important difference is the sign of $\sigma_{x y}$ which in our case comes out negative, hence opposite to the one in the $(3+1)$ dimensional setup. This is so, despite the fact that we have been careful in keeping all the conventions the same, and the sign in $\sigma_{x x}$ is positive as well. ${ }^{6}$

\footnotetext{
${ }^{6}$ Yet, notice a change of sign for very low values of $|E| / m^{2}$ approaching the Floquet condensates. It would be interesting to know if the configuration is unstable in this region and would decay to Minkowski embeddings.
} 
The region in phase space where a significant photoelectric Hall conductivity is obtained is rather small. It happens for values of the parameters in the region of $\Omega / m \leq 2$ and also small $|E| / m^{2} \leq 0.6$. This corresponds roughly to the close vicinity of the first dome to the left of the critical value $\Omega_{c}=1.496$ in figure 12 .

\subsection{Massless limit}

When the unperturbed embedding of the probe brane in massless, i.e., when $w=0$, the equations for $\delta w$ and $\delta \vec{c}$ decouple and we can take safely $\delta w=0$. The unperturbed configuration in this case is the analytic solution discussed in section 5.1. The equations of motion for $\delta c_{x}$ and $\delta c_{y}$ greatly simplify for this solution. Amazingly, the general solution of these coupled equations can be obtained analytically, as shown in detail in appendix D. Using these results one can extract the exact value of the conductivity matrices, which turn out to be

$$
\boldsymbol{\sigma}(\omega)=c \mathbb{I}, \quad \boldsymbol{\sigma}^{+}(\omega)=\boldsymbol{\sigma}^{-}(\omega)=0 .
$$

with $c=1$. As mentioned before, this analytical result controls the limits in the previous sections where $|E| / m^{2}$ and $\Omega / m$ both become very large.

Of course, the coefficient $c$ in eq. (6.28) is not equal to 1 . Using (2.24) it is easy to derive the correctly normalised physical conductivity, relating the physical electric field and the physical current, as

$$
\boldsymbol{\sigma}(\omega)=\frac{\sqrt{2} N_{f} N_{c}}{\pi \sqrt{\lambda}} \mathbb{I} .
$$

Up to a convention factor of $\sqrt{2}$ this result matches the one obtained in [50], see eq. (5.8), were the observation is made that this conductivity is nothing else than $1 / g^{2}$ in terms of the effective defect field theory gauge coupling on the D5-brane. This normalisation should affect all the conductivities obtained previously in this paper.

\section{Summary and outlook}

In this paper we employed holographic techniques to analyze the response of a $(2+1)$ dimensional gauge theory to an external rotating electric field. The system can be in two phases (insulator and conductor) depending on the type of embedding of the probe brane. In agreement with the $(3+1)$-dimensional results of $[26,27]$ we have found that for certain frequencies the driving field resonates with the vector meson excitations of the gauge theory and gives rise to zero field Floquet resonances. The lobbed structure shown in figure 2 may be a universal feature.

Transport properties are an important benchmark, and they exhibit substantial differences in the two dimensionalities. In $(2+1)$ dimensions, the massless case turns out to be trivial. In the massive situation, however, the conductivity tensor exhibits a rich structure. We have investigated the $\mathrm{AC}$ and $\mathrm{DC}$ conductivities, paying special attention to the first resonant frequency $\Omega=\Omega_{c}=1.4965$ which is close to the meson mass frequency. In this situation the system creates efficiently charged carriers and holes. In experimental setups, the asymmetry in the electron-hole pairs for different chiralities gives rise to the so called 
"optically induced valley polarization". This might be the closest physical realization to our setup at the resonant point. Weather the double peak structure observed in Fig: 11 may have some meaning in this direction is something that deserves further scrutiny. Otherwise, it would probably mean this model is too crude to capture the chiral asymmetry in the splitting of the Dirac point.

Many distinguishing features are present for configurations approaching the critical line separating the conducting from the isolating phase. The strong enhancement of the oscillations deforming into sharp peaks is one such. These peaks seem to point to bound states that are shifted from the meson masses. At this point, a very relevant question is that of stability of the embeddings. The exact point where, say, Black Hole embeddings decay into Minkowski embeddings and viceversa is a problem that can be addressed in equilbrium thermodynamics, and has a practical answer in terms of the equal area law. Here however, the similar tools are not available from a would be periodic thermodynamics [54], and this certainly an important aspect that will need further study in the future.

Let us now discuss possible related lines of research for the future. An interesting avenue would be to complete the phase diagram of the D3-D5 model by studying the system at (real) non-zero temperature and/or finite chemical potential. Moreover, we have treated the system in the quenched approximation. Dealing with the backreaction of the D5-branes is, in general, a tough problem. However, we could adopt some approximation, as it is done in the smearing approach (see [41] for a review). Interestingly, the smeared backreacted D3-D5 background has been obtained in [42] (see also [43-45]) and one could try to generalize these results to the case in which an external rotating electric field is acting on the flavor brane.

The comparison of our results with the D3-D7 model points towards some universality in the phase space. To strengthen this point, a natural strategy is to extend the study to other brane setups. Most prominently, the $(2+1)$ dimensional ABJM model is an example of superconformal QFT with a well stablished gravity dual [46]. The flavor branes in this case are D6-branes [47, 48] (see [49] for an analysis of the thermodynamics of the flavor branes in ABJM). The ABJM supergravity background has a non-trivial topological structure wich allows to switch on fluxes of the gauge fields and realize the quantum Hall effect [51]. This suggests that it could be the right place to find non-trivial topological effects induced by an external driving in an holographic setup like the ones we are missing here.

Another direction worth pushing is to consider other types of drivings. The brane picture of gauge theories allow for several possibilities. For example, instead of an electric field we could consider a magnetic field, or let the brane oscillate periodically. Actually, this last scenario has been considered in [52] in connection with the realization of the chiral magnetic effect in holography. It would be good to revisit this setup and search for possible stable non-equilibrium phases and condensates. The natural expectation is to observe resonances with the other type of mesons of the theory, like scalar mesons. 


\section{Acknowledgments}

We are indebted to Keiju Murata for many patient clarifications about his work. Also we would like to thank Yago Bea and Javier Tarrío for discussions and an early collaboration in the initial stages of this project. Special thanks also go to Takaaki Ishii inspiring conversations, and to Takashi Oka for insightful remarks on the physical interpretation of our results.

This work was supported by MINECO FPA2017- 84436-P, Xunta de Galicia ED431C 2017/07, Xunta de Galicia (Centro singular de investigación de Galicia accreditation 20192022) and the European Union (European Regional Development Fund - ERDF "María de Maeztu" Units of Excellence MDM- 2016-0692, and the Spanish Research State Agency. The work of A.G. has been supported by the Xunta de Galicia action under the grant ED481A-2019/054.

\section{A Regularity conditions}

In this appendix we derive the regularity conditions for black hole embeddings. The righthand side of the equations (2.17) must vanish at the pseudohorizon $\rho=\rho_{c}$ in order to avoid the potential singularity at this point. These conditions yield the following three equations

$$
\begin{aligned}
-\rho_{c}\left(\Omega+2 \rho_{c} b_{1}\right)\left(1+b_{1}^{2}+w_{1}^{2}\right)+\Omega b_{0}^{2}\left(\rho_{c}-2 b_{0} b_{1}\right) \chi_{1}^{2} & =0, \\
\rho_{c}\left(\rho_{c} w_{1}-w_{0}\right)\left(1+b_{1}^{2}+w_{1}^{2}\right)+\Omega b_{0}^{3} w_{1} \chi_{1}^{2} & =0, \\
\rho_{c}^{2}\left(-1+b_{1}^{2}+w_{1}^{2}\right)-\rho_{c}\left(2 w_{0} w_{1}-\Omega b_{1}\right)+\Omega b_{0}^{3} \chi_{1}^{2} & =0 .
\end{aligned}
$$

From the first equation in (A.1), we get

$$
\chi_{1}^{2}=\frac{\rho_{c}\left(\Omega+2 \rho_{c} b_{1}\right)\left(1+b_{1}^{2}+w_{1}^{2}\right)}{\Omega b_{0}^{2}\left(\rho_{c}-2 b_{0} b_{1}\right)} .
$$

Using this result in the second equation in (A.1) we arrive at the following relation between $b_{1}$ and $w_{1}$

$$
b_{1}=\frac{\Omega}{2 w_{0}} \frac{\rho_{c} w_{0}-\left(2 \rho_{c}^{2}+w_{0}^{2}\right) w_{1}}{\rho_{c}^{2}+w_{0}^{2}} .
$$

Let us next plug (A.2) into the third condition in (A.1). We get

$$
-w_{0} b_{1}^{2}+\left(4 \rho_{c} b_{0}+\Omega \rho_{c}+4 w_{0} b_{0} w_{1}\right) b_{1}+w_{0}^{2}-2 \rho_{c} w_{0} w_{1}+2\left(\rho_{c}^{2}+w_{0}^{2}\right) w_{1}^{2}=0 .
$$

Using now (A.3) we arrive at the following quadratic equation for $w_{1}$

$$
\left[1+\frac{\Omega^{2}\left(\rho_{c}^{2}+w_{0}^{2}\right)}{4\left(\rho_{c}^{2}+w_{0}^{2}\right)^{2}+\Omega^{2} \rho_{c}^{2}}\right] w_{1}^{2}+\frac{2 \rho_{c}}{w_{0}} w_{1}-1=0 .
$$

Let us write the solution of (A.5). First of all we define the quantities $s$ and $D$ as

$$
\begin{aligned}
s & \equiv \sqrt{\left[4\left(\rho_{c}^{2}+w_{0}^{2}\right)+\Omega^{2}\right]\left[4\left(\rho_{c}^{2}+w_{0}^{2}\right)^{2}+\Omega^{2} \rho_{c}^{2}\right]}, \\
D & \equiv 4\left(\rho_{c}^{2}+w_{0}^{2}\right)^{2}+\Omega^{2}\left(2 \rho_{c}^{2}+w_{0}^{2}\right) .
\end{aligned}
$$


Then, we can write

$$
w_{1}=\frac{1}{w_{0} D}\left[-4 \rho_{c}\left(\rho_{c}^{2}+w_{0}^{2}\right)^{2}-\Omega^{2} \rho_{c}^{3}+\left(\rho_{c}^{2}+w_{0}^{2}\right) s\right] .
$$

Let us now analyze some limiting cases of the relations found above.

\section{A.1 Large frequency}

Let us expand in powers of $1 / \Omega$ for large frequency $\Omega$. Taking the limit $\Omega \rightarrow \infty$ in (A.6) we get that $s \approx \rho_{c} \Omega^{2}$. and $D \approx\left(w_{0}^{2}+2 \rho_{c}^{2}\right) \Omega^{2}$. Plugging these values in (A.7) we get that $w_{1}$ is given at leading order by

$$
w_{1}=\frac{\rho_{c} w_{0}}{2 \rho_{c}^{2}+w_{0}^{2}} .
$$

Using this value of $w_{1}$ to evaluate the r.h.s. of (A.3) we get that $b_{1} \rightarrow 0$ as $\Omega \rightarrow \infty$. Actually, one can prove that

$$
b_{1}=-\frac{w_{0}^{2}\left(\rho_{c}^{2}+w_{0}^{2}\right)}{\rho_{c}\left(2 \rho_{c}^{2}+w_{0}^{2}\right)} \frac{1}{\Omega} .
$$

Taking into account the values of $b_{1}$ and $w_{1}$ found in this large $\Omega$ limit, we get the following value of $\chi^{\prime}$ at leading order

$$
\chi_{1}=\frac{\Omega}{\sqrt{\rho_{c}^{2}+w_{0}^{2}}} \frac{\sqrt{4 \rho_{c}^{2}+w_{0}^{2}}}{2 \rho_{c}^{2}+w_{0}^{2}} .
$$

\section{A.2 Small frequency}

When $\Omega \rightarrow 0$ the quantities $s$ and $D$ attain the values

$$
s \approx 4\left(\rho_{c}^{2}+w_{0}^{2}\right)^{\frac{3}{2}}, \quad D \approx 4\left(\rho_{c}^{2}+w_{0}^{2}\right)^{2}, \quad(\Omega \rightarrow 0),
$$

and one can verify readily that

$$
w_{1} \rightarrow \frac{1}{w_{0}}\left[-\rho_{c}+\sqrt{\rho_{c}^{2}+w_{0}^{2}}\right]
$$

\section{B Small mass solutions}

We now consider a small perturbation around the analytic massless solution of section 5.1. We write $b(\rho)$ and $w(\rho)$ as

$$
b(\rho)=b_{0}+\beta(\rho), \quad w(\rho)=\lambda(\rho),
$$

and we consider the equations of motion (2.17) at first-order in $\beta$ and $\lambda$. Using (2.18) to eliminate $\chi^{\prime}$, we arrive at the following decoupling linear equations for $\lambda(\rho)$ and $\beta(\rho)$

$$
\begin{array}{r}
\rho^{2}\left(\rho^{4}-q\right) \lambda^{\prime \prime}+2 \rho\left(\rho^{4}+q\right) \lambda^{\prime}-2 q \lambda=0 \\
\rho^{2}\left(\rho^{4}-q\right) \beta^{\prime \prime}+2 \rho\left(\rho^{4}+q\right) \beta^{\prime}+4 \Omega^{2} \frac{\rho^{6}}{\rho^{4}-q} \beta=0 .
\end{array}
$$


(We are assuming that $q>0$ ). Let us consider from now on the equation for $\lambda$ in (B.3). This equation can be solved analytically in terms of hypergeometric functions

$$
\lambda(\rho)=c_{1} \rho F\left(\frac{1}{4}, \frac{1}{2} ; \frac{3}{4} ; \frac{\rho^{4}}{q}\right)+c_{2} \rho^{2} F\left(\frac{1}{2}, \frac{3}{4} ; \frac{5}{4} ; \frac{\rho^{4}}{q}\right),
$$

where $c_{1}$ and $c_{2}$ are constants. The hypergeometric functions written in (B.4) have a logarithmic singularity as we approach the pseudohorizon at $\rho=q^{\frac{1}{4}}$. Indeed, from the general equation

$$
F(\alpha, \beta ; \alpha+\beta ; z) \approx-\frac{\Gamma(\alpha+\beta)}{\Gamma(\alpha) \Gamma(\beta)} \log (1-z), \quad \text { as } z \rightarrow 1^{-},
$$

we get

$$
\lambda(\rho) \approx-\frac{q^{\frac{1}{4}}}{\sqrt{\pi}}\left[c_{1} \frac{\Gamma\left(\frac{3}{4}\right)}{\Gamma\left(\frac{1}{4}\right)}+c_{2} q^{\frac{1}{4}} \frac{\Gamma\left(\frac{5}{4}\right)}{\Gamma\left(\frac{3}{4}\right)}\right] \log \left(1-\frac{\rho^{4}}{q}\right), \quad \text { as } \rho \rightarrow q^{\frac{1}{4}} .
$$

The absence of this logarithmic singularity imposes the following ratio between the constants $c_{1}$ and $c_{2}$

$$
\frac{c_{1}}{c_{2}}=-\frac{q^{\frac{1}{4}}}{4}\left[\frac{\Gamma\left(\frac{1}{4}\right)}{\Gamma\left(\frac{3}{4}\right)}\right]^{2} .
$$

Let us now look at the UV behavior $\rho \rightarrow \infty$. In general, for large $z$, one has

$$
F(\alpha, \beta ; \alpha+\beta ; z) \approx \Gamma(\alpha+\beta)\left[e^{-i \pi \alpha} \frac{\Gamma(\beta-\alpha)}{\Gamma^{2}(\beta)} z^{-\alpha}+e^{-i \pi \beta} \frac{\Gamma(\alpha-\beta)}{\Gamma^{2}(\alpha)} z^{-\beta}\right] .
$$

Applying this last equation to our case, we get the UV behavior of the two functions in (B.4)

$$
\begin{gathered}
\rho F\left(\frac{1}{4}, \frac{1}{2} ; \frac{3}{4} ; \frac{\rho^{4}}{q}\right) \approx q^{\frac{1}{4}} \Gamma\left(\frac{3}{4}\right)\left[\frac{e^{-\frac{i \pi}{4}}}{\pi} \Gamma\left(\frac{1}{4}\right)+q^{\frac{1}{4}} \frac{e^{-\frac{i \pi}{2}} \Gamma\left(-\frac{1}{4}\right)}{\Gamma^{2}\left(\frac{1}{4}\right)} \frac{1}{\rho}\right], \\
\rho^{2} F\left(\frac{1}{2}, \frac{3}{4} ; \frac{5}{4} ; \frac{\rho^{4}}{q}\right) \approx q^{\frac{1}{4}} \Gamma\left(\frac{5}{4}\right)\left[q^{\frac{1}{4}} e^{-\frac{i \pi}{2}} \frac{\Gamma\left(\frac{1}{4}\right)}{\Gamma^{2}\left(\frac{3}{4}\right)}+q^{\frac{1}{2}} \frac{e^{-\frac{3 i \pi}{2}}}{\pi} \Gamma\left(-\frac{1}{4}\right) \frac{1}{\rho}\right] .
\end{gathered}
$$

Therefore, the coefficient of the leading term in $\lambda(\rho)$ as $\rho \rightarrow \infty$ is

$$
q^{\frac{1}{4}} c_{1} e^{-\frac{i \pi}{4}} \frac{\Gamma\left(\frac{3}{4}\right) \Gamma\left(\frac{1}{4}\right)}{\pi}+q^{\frac{1}{2}} c_{2} e^{-\frac{i \pi}{2}} \Gamma\left(\frac{5}{4}\right) \frac{\Gamma\left(\frac{1}{4}\right)}{\Gamma^{2}\left(\frac{3}{4}\right)} .
$$

The imaginary part of this leading term in the UV is zero if the ratio of $c_{1}$ and $c_{2}$ is

$$
\frac{c_{1}}{c_{2}}=-\frac{q^{\frac{1}{4}}}{4} \frac{\sqrt{2} \Gamma\left(\frac{5}{4}\right)}{\Gamma^{3}\left(\frac{3}{4}\right)}
$$

which can be seen to be equivalent to (B.7). If this ratio holds, the leading term $\lambda(\rho=\infty)$ is finite and given by

$$
m \equiv q^{\frac{1}{4}} c_{1} \frac{\Gamma\left(\frac{3}{4}\right) \Gamma\left(\frac{1}{4}\right)}{\sqrt{2} \pi}=q^{\frac{1}{4}} c_{1} .
$$


Let us now look at the UV subleading term, which is of the form $\mathcal{C} / \rho$. The coefficient $\mathcal{C}$ (i.e., the condensate), is given by

$$
\mathcal{C}=q^{\frac{1}{2}} c_{1} e^{-\frac{i \pi}{2}} \frac{\Gamma\left(\frac{3}{4}\right) \Gamma\left(-\frac{1}{4}\right)}{\Gamma^{2}\left(\frac{1}{4}\right)}+q^{\frac{3}{4}} c_{2} e^{-\frac{3 i \pi}{4}} \frac{\Gamma\left(\frac{5}{4}\right) \Gamma\left(-\frac{1}{4}\right)}{\pi} .
$$

The imaginary part of $\mathcal{C}$ vanishes if

$$
\frac{c_{1}}{c_{2}}=-q^{\frac{1}{4}} \frac{\Gamma\left(\frac{5}{4}\right) \Gamma^{2}\left(\frac{1}{4}\right)}{\sqrt{2} \pi \Gamma\left(\frac{3}{4}\right)},
$$

which again can be proven to be equivalent to (B.7). Then, the condensate $\mathcal{C}$ is given by

$$
\mathcal{C}=-q^{\frac{3}{4}} c_{2} \frac{\Gamma\left(\frac{5}{4}\right) \Gamma\left(-\frac{1}{4}\right)}{\sqrt{2} \pi}=q^{\frac{3}{4}} c_{2}
$$

By combining (B.15), (B.12) and (B.7) we can obtain the relation between $\mathcal{C}$ and $m$

$$
\mathcal{C}=-q^{\frac{1}{4}}\left[\frac{2 \Gamma\left(\frac{3}{4}\right)}{\Gamma\left(\frac{1}{4}\right)}\right]^{2} m
$$

Therefore, in this small mass regime, the condensate is proportional to the mass with a coefficient which depends on $q^{\frac{1}{4}}=\sqrt{\Omega b_{0}}$. Notice that we can write the solution for $w=\lambda$ in terms of the mass and condensante as

$$
w(\rho)=\frac{m}{|q|^{\frac{1}{4}}} \rho F\left(\frac{1}{4}, \frac{1}{2} ; \frac{3}{4} ; \frac{\rho^{4}}{|q|}\right)+\frac{\mathcal{C}}{|q|^{\frac{3}{4}}} \rho^{2} F\left(\frac{1}{2}, \frac{3}{4} ; \frac{5}{4} ; \frac{\rho^{4}}{|q|}\right),
$$

where $\mathcal{C}$ is related to $m$ as in (B.16). We can also obtain the value of the embedding function at the pseudohorizon in terms of the mass

$$
w\left(\rho=\sqrt{\Omega b_{0}}\right)=\frac{\Gamma^{2}\left(\frac{3}{4}\right)}{\sqrt{2 \pi}} m .
$$

Let us now integrate the equation in (B.3) for the fluctuation $\beta$ of the electric field. This equation can be solved analytically,. In order to write this solution, let us define the following hatted quantities

$$
\hat{\rho}=\frac{\rho}{q^{\frac{1}{4}}}, \quad \hat{\Omega}=\frac{\Omega}{q^{\frac{1}{4}}},
$$

and the two functions $F(\hat{\rho})$ and $G(\hat{\rho})$ as

$$
F(\hat{\rho}) \equiv \hat{\Omega} \arctan (\hat{\rho}), \quad G(\hat{\rho}) \equiv \frac{\hat{\Omega}}{2} \log \frac{\hat{\rho}-1}{\hat{\rho}+1} .
$$

Then, the general solution for the function $\beta$ is

$$
\beta(\hat{\rho})=c_{1} \cos [F(\hat{\rho})+G(\hat{\rho})]+c_{2} \sin [F(\hat{\rho})+G(\hat{\rho})],
$$


where $c_{1}$ and $c_{2}$ are real constants. In order to find $E$ and $j$ for this solution, we must study the UV behavior of the complex potential $c=b e^{i \chi}$. The equation for the perturbation $\delta c=c-b_{0} e^{-i \Omega / \rho}$ at first order is

$$
\rho^{2}\left(\rho^{4}-q\right) \delta c^{\prime \prime}+2\left(\rho\left(\rho^{4}+q\right)+i q \Omega\right) \delta c^{\prime}+\frac{\Omega^{2}}{\rho^{2}}\left(\rho^{4}+q-4 i \rho \frac{q}{\omega}\right) \delta c=0 .
$$

It is difficult to solve this equation directly. However, the equation for $\delta \chi=\chi+\frac{\Omega}{\rho}$ can be obtained form (2.18) and is rather simple

$$
\delta \chi^{\prime}=-\frac{2 \rho^{2} \Omega}{b_{0}\left(\rho^{4}-q\right)} \beta .
$$

Notice that $\delta c$ is related to $\beta$ and $\delta \chi$ as

$$
\delta c=e^{-i \frac{\Omega}{\rho}}\left(\beta+i b_{0} \delta \chi\right)
$$

One can check that the second equation in (B.3), together with (B.23), imply (B.22). Moreover, since we know the solution for $\beta$ we can directly integrate (B.23). Indeed, let us write the solution (B.21) in terms of complex exponentials as

$$
\beta=d e^{-i(F(\hat{\rho})+G(\hat{\rho}))}+d^{*} e^{i(F(\hat{\rho})+G(\hat{\rho}))},
$$

with $d$ being a complex constant. Then, we can show that $\delta \chi$ is

$$
\delta \chi=-\frac{i d}{b_{0}} e^{-i(F(\hat{\rho})+G(\hat{\rho}))}+\frac{i d^{*}}{b_{0}} e^{i(F(\hat{\rho})+G(\hat{\rho}))}+\lambda,
$$

with $\lambda \in \mathbb{R}$. Plugging $\beta$ and $\delta \chi$ on the right-hand side of (B.24), we get

$$
\delta c=2 d e^{-i \frac{\Omega}{q^{1 / 4}}\left(\frac{q^{1 / 4}}{\rho}+\arctan \left(\rho / q^{1 / 4}\right)+\frac{1}{2} \log \frac{\rho-q^{1 / 4}}{\rho+q^{1 / 4}}\right)}+i b_{0} \lambda e^{-i \frac{\Omega}{\rho}} .
$$

Notice that the function multiplying $d$ is an outgoing wave, whereas that multiplying $\lambda$ is an incoming wave. It is also interesting to notice that the first order variation of the Joule heating $q$ is zero for this solution, as it can be checked by using (2.16).

\section{Embeddings in the linearized approximation}

In this appendix we study in detail the three types of embeddings in the linearized approximation of section 5.2 (valid for large driving frequency $\Omega$ or small electric field $E$ ).

\section{C.1 Minkowski embeddings}

To obtain the Minkowski embeddings that reach $\rho=0$ we impose regularity of the function $c(\rho)$ at $\rho=0$. This implies that the constant $c_{2}$ must vanish and, therefore the gauge field $c(\rho)$ is given by

$$
c(\rho)=c_{1} g_{1}(\rho)=c_{1} \frac{\sqrt{1+\rho^{2}}}{\rho} \sin \left(\sqrt{1+\Omega^{2}} \arctan (\rho)\right) .
$$


From the boundary expansion (2.22) we find the electric field and current hence

$$
\begin{aligned}
E & =-i c_{1} \Omega \sin \left(\frac{\pi}{2} \sqrt{1+\Omega^{2}}\right), \\
j & =-c_{1} \sqrt{1+\Omega^{2}} \cos \left(\frac{\pi}{2} \sqrt{1+\Omega^{2}}\right) .
\end{aligned}
$$

Eliminating the constant $c_{1}$ we obtain the current in terms of the electric field $E$

$$
j=-\frac{i E}{\Omega} \sqrt{1+\Omega^{2}} \cot \left(\frac{\pi}{2} \sqrt{1+\Omega^{2}}\right) .
$$

We can also eliminate $c_{1}$ in (C.1) using (C.2)

$$
c(\rho)=\frac{i E}{\Omega \sin \left[\frac{\pi}{2} \sqrt{1+\Omega^{2}}\right]} \frac{\sqrt{1+\rho^{2}}}{\rho} \sin \left[\sqrt{1+\Omega^{2}} \arctan \rho\right] .
$$

Let us now look at the embedding function $\delta w$ given in (5.21). Imposing $\delta w(\rho=0)=0$ fixes the value of the integration constant $c_{3}$

$$
c_{3}=2 \Omega^{2}\left|c_{1}\right|^{2} \int_{0}^{\infty} d s \frac{s}{\left(s^{2}+1\right)^{3}} g_{1}^{2}(s) .
$$

Then, the resulting $\delta w(\rho)$ can be written as

$$
\begin{aligned}
\delta w(\rho)= & 2 \Omega^{2}\left|c_{1}\right|^{2} \int_{0}^{\rho} d s \frac{\sin ^{2}\left[\sqrt{1+\Omega^{2}} \arctan (s)\right]}{s\left(s^{2}+1\right)^{2}}- \\
& -\frac{2 \Omega^{2}\left|c_{1}\right|^{2}}{\rho} \int_{0}^{\rho} d s \frac{\sin ^{2}\left[\sqrt{1+\Omega^{2}} \arctan (s)\right]}{\left(s^{2}+1\right)^{2}} .
\end{aligned}
$$

From the UV expansion (2.23) we find the mass and the condensate

$$
\begin{aligned}
& m=1+2 \Omega^{2}\left|c_{1}\right|^{2} \int_{0}^{\infty} d s \frac{\sin ^{2}\left[\sqrt{1+\Omega^{2}} \arctan (s)\right]}{s\left(s^{2}+1\right)^{2}} \\
& \mathcal{C}=-2 \Omega^{2}\left|c_{1}\right|^{2} \int_{0}^{\infty} d s \frac{\sin ^{2}\left[\sqrt{1+\Omega^{2}} \arctan (s)\right]}{\left(s^{2}+1\right)^{2}} .
\end{aligned}
$$

In terms of the electric field at the boundary, using (C.2) we obtain

$$
\begin{aligned}
& m=1+\frac{2|E|^{2}}{\sin ^{2}\left(\frac{\pi}{2} \sqrt{1+\Omega^{2}}\right)} \int_{0}^{\infty} d s \frac{\sin ^{2}\left[\sqrt{1+\Omega^{2}} \arctan (s)\right]}{s\left(s^{2}+1\right)^{2}} \\
& \mathcal{C}=-\frac{|E|^{2}}{4 \sin ^{2}\left(\frac{\pi}{2} \sqrt{1+\Omega^{2}}\right)}\left(\pi+\frac{\sin \left(\pi \sqrt{1+\Omega^{2}}\right)}{\Omega^{2} \sqrt{1+\Omega^{2}}}\right)
\end{aligned}
$$

To find the resonant frecuencies of the Floquet condensates we impose that $E=0$ in (C.2), which means that the $\Omega$ 's must satisfy

$$
\sqrt{1+\Omega_{n}^{2}}=2(n+1),
$$

for $n=0,1,2, \cdots$. This gives the following discrete set

$$
\Omega_{n}=2 \sqrt{\left(n+\frac{1}{2}\right)\left(n+\frac{3}{2}\right)},
$$

which are the same as the masses of the vector mesons in the D3-D5 model [32]. 


\section{C.2 Black hole embeddings}

We now determine $c(\rho)$ for a black hole embedding by imposing the appropriate in-falling boundary condition at the pseudohorizon. Now the phase $\chi(\rho)$ is not constant and, in fact, $\chi^{\prime}$ is related to the Joule heating $q$. To obtain this relation in the linearized approximation, let us take into account that $c c^{\prime *}-c^{*} c^{\prime}=-2 i \chi^{\prime} b^{2}$. Since the lagrangian density $\mathcal{L}=\rho^{2}$ in the linear approximation, we get from (2.16) that

$$
\chi^{\prime}(\rho)=\frac{q}{\Omega} \frac{1}{\rho^{2} b^{2}(\rho)} .
$$

Let us now take $\rho=\rho_{c}$ in this last expression and use (3.1) and (3.5) to relate $b_{0}=b\left(\rho_{c}\right)$ and $q$ to $w_{0}=w\left(\rho_{c}\right)$ and $\rho_{c}$. We immediately get

$$
\chi^{\prime}\left(\rho_{c}\right)=\frac{\Omega}{w_{0}^{2}+\rho_{c}^{2}},
$$

which is an initial condition for $\chi(\rho)$. We begin by introducing a new variable $y$, related to $\rho$ as $^{7}$

$$
y=\arctan (\rho),
$$

and let $y_{c}$ be the $y$ coordinate of the pseudohorizon $y_{c}=\arctan \left(\rho_{c}\right)$. In terms of the variable $y$, the initial condition (C.13), for $w_{0}=1$, takes the form

$$
\left.\frac{d \chi}{d y}\right|_{y=y_{c}}=\Omega .
$$

We will impose the following infalling condition at the pseudohorizon

$$
\left.\frac{d c}{d y}\right|_{y=y_{c}}=i \Omega c\left(y_{c}\right) .
$$

Taking into account that, in general

$$
\frac{d c}{d y}=\left[i \frac{d \chi}{d y}+\frac{1}{b} \frac{d b}{d y}\right] c,
$$

this is equivalent to requiring that

$$
\left.\frac{d b}{d y}\right|_{y=y_{c}}=0
$$

or, equivalently, that the infalling frequency is real. To simplify the calculation, let us now introduce a new complex field

$$
\psi(y)=\frac{\rho}{\sqrt{1+\rho^{2}}} c(\rho) .
$$

\footnotetext{
${ }^{7}$ The coordinate $y$ is nothing but the tortoise coordinate $\rho_{*}$ in this linear approximation. Indeed, in this case the function $B(\rho)$ defined in $(3.13)$ is $B(\rho) \approx 1 /\left(1+\rho^{2}\right)$ and (3.12) is easily integrated to give $\rho_{*}=\arctan y$, as claimed.
} 
As $c=\psi(y) / \sin y$, the infalling boundary condition (C.16) takes the following form in terms of $\psi$

$$
\left.\frac{d \psi}{d y}\right|_{y=y_{c}}=\left(i \Omega+\cot y_{c}\right) \psi\left(y_{c}\right)
$$

Taking into account that $\cot y_{c}=1 / \rho_{c}$, we finally get

$$
\left.\frac{d \psi}{d y}\right|_{y=y_{c}}=\left(i \Omega+\frac{1}{\rho_{c}}\right) \psi\left(y_{c}\right) .
$$

The general solution (5.15) can be written as

$$
\psi(y)=d_{1} e^{i \bar{\Omega} y}+d_{2} e^{-i \bar{\Omega} y},
$$

where $d_{1}$ and $d_{2}$ are complex constants and $\bar{\Omega}$ is defined as

$$
\bar{\Omega} \equiv \sqrt{1+\Omega^{2}}
$$

We now impose the pseudohorizon boundary condition (C.16). It is easy to demonstrate that (C.16) is satisfied if the ratio of the constants $d_{1}$ and $d_{2}$ is given by

$$
\frac{d_{1}}{d_{2}}=\frac{(\bar{\Omega}+\Omega) \rho_{c}-i}{(\bar{\Omega}-\Omega) \rho_{c}+i} e^{-2 i \bar{\Omega} y_{c}}
$$

To rewrite this condition in a more convenient form, define the phases $\Lambda_{c}$ and $\bar{\Lambda}_{c}$

$$
\Lambda_{c} \equiv \arctan \left[\frac{\bar{\Omega}-\Omega}{\rho_{c}}\right], \quad \bar{\Lambda}_{c} \equiv \arctan \left[\frac{\bar{\Omega}+\Omega}{\rho_{c}}\right]
$$

in terms of which we can write

$$
\begin{aligned}
& (\bar{\Omega}+\Omega) \rho_{c}+i=\sqrt{(\bar{\Omega}+\Omega)^{2} \rho_{c}^{2}+1} e^{i \Lambda_{c}}, \\
& (\bar{\Omega}-\Omega) \rho_{c}+i=\sqrt{(\bar{\Omega}-\Omega)^{2} \rho_{c}^{2}+1} e^{i \bar{\Lambda}_{c}}
\end{aligned}
$$

and $d_{1} / d_{2}$ takes the form

$$
\frac{d_{1}}{d_{2}}=\frac{\sqrt{(\bar{\Omega}+\Omega)^{2} \rho_{c}^{2}+1}}{\sqrt{(\bar{\Omega}-\Omega)^{2} \rho_{c}^{2}+1}} e^{-2 i \bar{\Omega} y_{c}-i \Lambda_{c}-i \bar{\Lambda}_{c}} .
$$

It is now straightforward to relate the value of $\psi$ at $y=y_{c}$ with the constant $d_{1}$ and to obtain the value of $c$ at the pseudohorizon. We get

$$
c\left(y_{c}\right)=\frac{2 \bar{\Omega} \sqrt{1+\rho_{c}^{2}}}{\sqrt{(\bar{\Omega}+\Omega)^{2} \rho_{c}^{2}+1}} d_{1} e^{i \bar{\Omega} y_{c}+i \Lambda_{c}}
$$

We choose the phase of $c\left(y_{c}\right)$ to vanish at $\rho=\rho_{c}$. This requirement determines the phase of $d_{1}$, which must be of the form

$$
d_{1}=\left|d_{1}\right| e^{-i \bar{\Omega} y_{c}-i \Lambda_{c}}
$$


Moreover, since

$$
\left|c\left(y_{c}\right)\right|=\frac{2 \bar{\Omega} \sqrt{1+\rho_{c}^{2}}}{\sqrt{(\bar{\Omega}+\Omega)^{2} \rho_{c}^{2}+1}}\left|d_{1}\right|,
$$

we can fulfill (3.1) with $w_{0}=1$ by choosing $\left|d_{1}\right|$ to be

$$
\left|d_{1}\right|=\frac{\sqrt{1+\rho_{c}^{2}}}{2 \bar{\Omega} \Omega} \sqrt{(\bar{\Omega}+\Omega)^{2} \rho_{c}^{2}+1} .
$$

The value of $\left|d_{2}\right|$ can be obtained from (C.27)

$$
\left|d_{2}\right|=\frac{\sqrt{1+\rho_{c}^{2}}}{2 \bar{\Omega} \Omega} \sqrt{(\bar{\Omega}-\Omega)^{2} \rho_{c}^{2}+1} .
$$

From these values of $\left|d_{1}\right|$ and $\left|d_{2}\right|$ one can readily check that the Joule heating $q$ is given by

$$
q=\Omega \bar{\Omega}\left(\left|d_{1}\right|^{2}-\left|d_{2}\right|^{2}\right)=\left(1+\rho_{c}^{2}\right) \rho_{c}^{2},
$$

in agreement with (3.5), as it should. Taking into account these results, we can write $d_{1}$ and $d_{2}$ as

$$
\begin{aligned}
& d_{1}=\frac{\sqrt{1+\rho_{c}^{2}}}{2 \bar{\Omega} \Omega} \sqrt{(\bar{\Omega}+\Omega)^{2} \rho_{c}^{2}+1} e^{-i \bar{\Omega} y_{c}-i \Lambda_{c}}=\frac{\sqrt{1+\rho_{c}^{2}}}{2 \bar{\Omega} \Omega}\left[(\bar{\Omega}+\Omega) \rho_{c}-i\right] e^{-i \bar{\Omega} y_{c}}, \\
& d_{2}=\frac{\sqrt{1+\rho_{c}^{2}}}{2 \bar{\Omega} \Omega} \sqrt{(\bar{\Omega}-\Omega)^{2} \rho_{c}^{2}+1} e^{i \bar{\Omega} y_{c}+i \bar{\Lambda}_{c}}=\frac{\sqrt{1+\rho_{c}^{2}}}{2 \bar{\Omega} \Omega}\left[(\bar{\Omega}-\Omega) \rho_{c}+i\right] e^{i \bar{\Omega} y_{c}},
\end{aligned}
$$

and the complexified gauge potential $c(y)$ is given by

$$
\begin{aligned}
c(y)= & \frac{\sqrt{1+\rho_{c}^{2}}}{2 \bar{\Omega} \Omega \sin y}\left[\sqrt{(\bar{\Omega}+\Omega)^{2} \rho_{c}^{2}+1} e^{i \bar{\Omega}\left(y-y_{c}\right)-i \Lambda_{c}}+\right. \\
& \left.+\sqrt{(\bar{\Omega}-\Omega)^{2} \rho_{c}^{2}+1} e^{-i \bar{\Omega}\left(y-y_{c}\right)+i \bar{\Lambda}_{c}}\right] .
\end{aligned}
$$

This expression can also be rewritten as

$$
c(y)=\frac{\sqrt{1+\rho_{c}^{2}}}{2 \bar{\Omega} \Omega \sin y}\left[\left[(\bar{\Omega}+\Omega) \rho_{c}-i\right] e^{i \bar{\Omega}\left(y-y_{c}\right)}+\left[(\bar{\Omega}-\Omega) \rho_{c}+i\right] e^{-i \bar{\Omega}\left(y-y_{c}\right)}\right] .
$$

From (C.36) we can obtain the phase $\chi$

$$
\tan \chi(y)=\frac{\rho_{c} \Omega \sin \left[\bar{\Omega}\left(y-y_{c}\right)\right]}{\rho_{c} \bar{\Omega} \cos \left[\bar{\Omega}\left(y-y_{c}\right)\right]+\sin \left[\bar{\Omega}\left(y-y_{c}\right)\right]} .
$$

Finally, we can expand this result near $\rho=\infty$ to obtain the electric field $E$

$$
\begin{aligned}
E= & -i \frac{\sqrt{1+\rho_{c}^{2}}}{2 \bar{\Omega}}\left[\sqrt{(\bar{\Omega}+\Omega)^{2} \rho_{c}^{2}+1} e^{-i \bar{\Omega}\left(y_{c}-\frac{\pi}{2}\right)-i \Lambda_{c}}+\right. \\
& \left.+\sqrt{(\bar{\Omega}-\Omega)^{2} \rho_{c}^{2}+1} e^{i \bar{\Omega}\left(y_{c}-\frac{\pi}{2}\right)+i \bar{\Lambda}_{c}}\right]
\end{aligned}
$$


and the current $j$

$$
\begin{aligned}
& j=-i \frac{\sqrt{1+\rho_{c}^{2}}}{2 \Omega}\left[\sqrt{(\bar{\Omega}+\Omega)^{2} \rho_{c}^{2}+1} e^{-i \bar{\Omega}\left(y_{c}-\frac{\pi}{2}\right)-i \Lambda_{c}}-\right. \\
& \left.-\sqrt{(\bar{\Omega}-\Omega)^{2} \rho_{c}^{2}+1} e^{i \bar{\Omega}\left(y_{c}-\frac{\pi}{2}\right)+i \bar{\Lambda}_{c}}\right] .
\end{aligned}
$$

As a check one can verify that these results reduce to the ones obtained for Minkowski embeddings when we take $\rho_{c}=y_{c}=0$.

\section{C.3 Critical embeddings}

According to our general analysis of section 3.3, the critical solutions satisfy $\Omega b(\rho=0)=$ $w_{0}^{2}$. Therefore, if we define the parameter $\epsilon$ as

$$
\epsilon \equiv \Omega b(\rho=0)
$$

these embeddings must satisfy $\epsilon=w_{0}^{2}$. In our linearized analysis we have $w_{0}=1$. Thus, we are tempted to describe the critical embeddings by means of the solution $g_{1}(\rho)$ of (C.1) with $\epsilon=1$. Indeed, contrary to the other solution $g_{2}(\rho)$, the function $g_{1}(\rho)$ is regular at $\rho=0$ and one can easily find $\epsilon$ as a function of $\Omega$ and the constant $c_{1}$

$$
\epsilon=\Omega c_{1} \sqrt{1+\Omega^{2}} .
$$

Plugging this relation in (C.1), we get $c(\rho)$ as

$$
c(\rho)=\frac{\epsilon}{\Omega \sqrt{1+\Omega^{2}}} g_{1}(\rho) .
$$

It turns out, however, that this linearized solution cannot describe accurately the critical embedding near $\rho=0$. Indeed, we found in section 3.3 that the $b$ field behaves linearly in $\rho$ around its value at $\rho=0$, whereas the function $g_{1}(\rho)$ behaves quadratically (see (5.18)). As argued in [27] for the analogous D3-D7 case, the critical solutions behave highly nonlinearly around $\rho=0$. Nevertheless the linear solution can describe rather accurately the true non-linear solution for $\rho \gg 1$ if we change the value of $\epsilon$ from its naive value $\epsilon=1$. To illustrate this we compare the linear analytic solution for $\epsilon=1 / 2$ with the numerical results. In figure 15 we plot the values of $c(\rho)$ calculated by these two procedures.

For a general value of $\epsilon$, the electric field $E$, the current $j$ and the condensate $\mathcal{C}$ in the linear approximation are

$$
\begin{aligned}
& E=-i \frac{\epsilon}{\sqrt{1+\Omega^{2}}} \sin \left(\frac{\pi}{2} \sqrt{1+\Omega^{2}}\right), \\
& j=-\frac{\epsilon}{\Omega} \cos \left(\frac{\pi}{2} \sqrt{1+\Omega^{2}}\right) \\
& \mathcal{C}=-\frac{1}{4} \frac{\epsilon^{2}}{1+\Omega^{2}}\left(\pi+\frac{\sin \left(\pi \sqrt{1+\Omega^{2}}\right)}{\Omega^{2} \sqrt{1+\Omega^{2}}}\right) .
\end{aligned}
$$




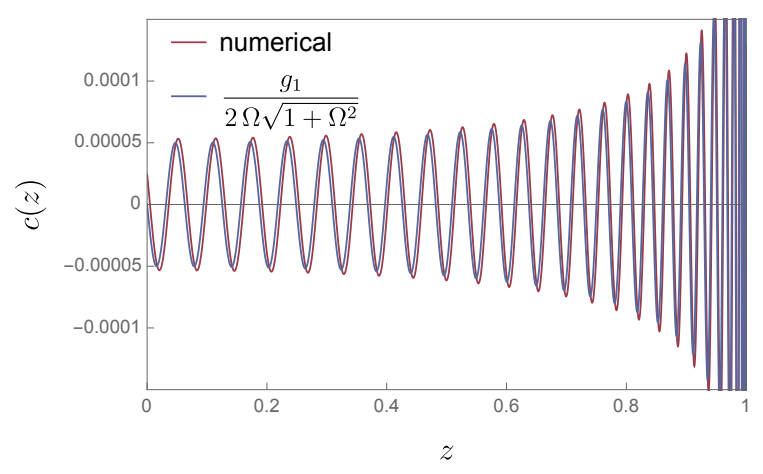

Figure 15. Comparison of the critical solution for $c$ obtained by numerically solving the non-linear full equation of motion and (C.42) for $\Omega=100, m=1$ and $\epsilon=\frac{1}{2}$, using $z^{2}=\frac{1}{1+\rho^{2}}$.
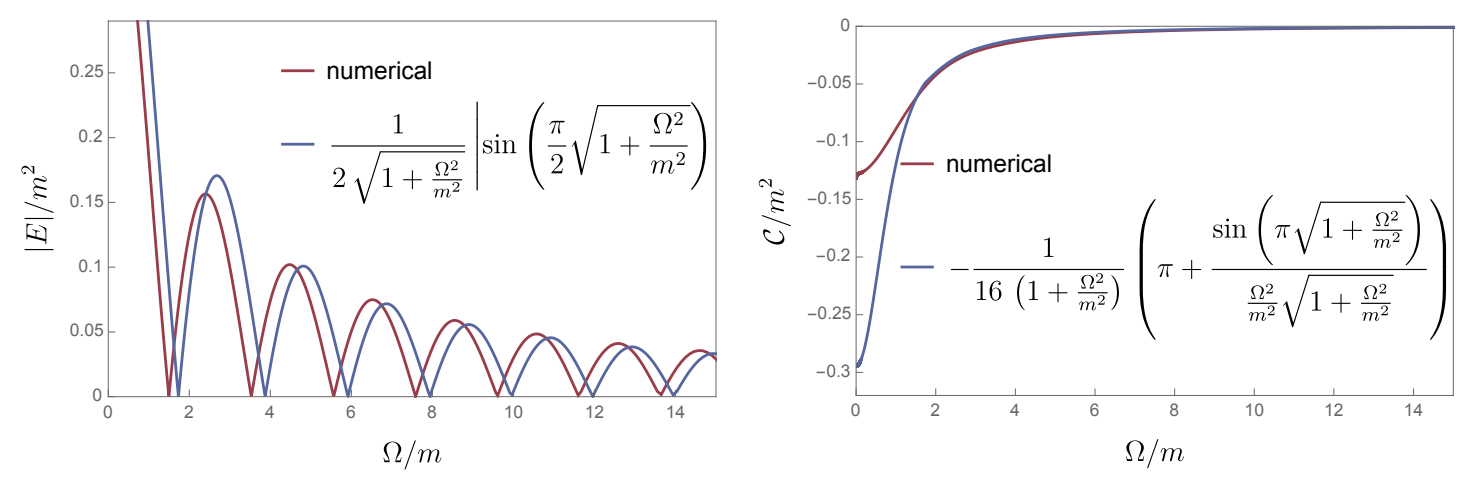

Figure 16. $E$ and $\mathcal{C}$ for critical solutions obtained numerically and using (C.43) with $\epsilon=1 / 2$.

In figure 16 we compare the approximate linear expressions (C.43) for $E$ and $\mathcal{C}$ (again for $\epsilon=1 / 2$ ) to the numerical result of the full non-linear calculation. A reasonable agreement for large $\Omega$ is found. The discrepancies can be attributed to the intrinsic non-linearity of the critical solutions. In particular, eq. (C.43) implies that the critical electric field decreases as $(\Omega / m)^{-1}$ for large $\Omega / m$. We can also use (C.43) to estimate the susceptibility parameter $\gamma_{x y}$ (defined in (4.4)) for critical embeddings:

$$
\gamma_{x y}=\frac{\operatorname{Im}\left(E j^{*}\right)}{|E|^{2}}=\frac{\sqrt{1+\Omega^{2}}}{\Omega} \cot \left(\frac{\pi}{2} \sqrt{1+\Omega^{2}}\right) .
$$

This expression explains qualitatively well the change of sign of $\gamma_{x y}$ pointed out in section 4 and reproduces quite accurately the numerical results of $\gamma_{x y}$ for large values of the driving frequency $\Omega$.

\section{Conductivities in the massless case}

In the massless case the fluctuations $\delta w$ of the embedding function decouple from those of the gauge field $\delta \vec{c}$. Therefore, since we are interested in computing conductivities, we can 
concentrate in studying the equations for $\delta c_{x}$ and $\delta c_{y}$. In order to write these equations in a more convenient form, let us define the following differential operators

$$
\begin{aligned}
& \mathcal{O}_{1} \equiv \partial_{t}^{2}+\frac{\rho^{4}\left(\rho_{c}^{4}-\rho^{4}\right)}{\rho_{c}^{4}+\rho^{4}} \partial_{\rho}^{2}-\frac{2 \rho_{c}^{4} \rho^{2}}{\rho_{c}^{4}+\rho^{4}} \partial_{t} \partial_{\rho}+\frac{4 \rho_{c}^{4} \rho}{\rho_{c}^{4}+\rho^{4}} \partial_{t}-2 \rho^{3} \partial_{\rho}, \\
& \mathcal{O}_{2} \equiv-2 \partial_{t}+\frac{2 \rho_{c}^{4} \rho}{\rho_{c}^{4}+\rho^{4}}\left(\rho \partial_{\rho}-2\right) .
\end{aligned}
$$

Then, $\delta c_{x}$ and $\delta c_{y}$ satisfy the following system of coupled second-order differential equations

$$
\left(\mathcal{O}_{1}-\Omega^{2}\right) \delta c_{x}+\Omega \mathcal{O}_{2} \delta c_{y}=0, \quad\left(\mathcal{O}_{1}-\Omega^{2}\right) \delta c_{y}-\Omega \mathcal{O}_{2} \delta c_{x}=0 .
$$

To decouple these equations, let us consider the following complex combinations of $\delta c_{x}$ and $\delta c_{y}$

$$
\eta(t, \rho) \equiv \delta c_{x}(t, \rho)+i \delta c_{y}(t, \rho), \quad \tilde{\eta}(t, \rho) \equiv \delta c_{x}(t, \rho)-i \delta c_{y}(t, \rho) .
$$

Notice that $\tilde{\eta}$ is not the complex conjugate of $\eta$ since $\delta c_{x}$ and $\delta c_{y}$ are not necessarily real. It is straightforward to verify that the equations for $\eta$ and $\tilde{\eta}$ are indeed decoupled and given by

$$
\left(\mathcal{O}_{1}-\Omega^{2}\right) \eta-i \Omega \mathcal{O}_{2} \eta=0, \quad\left(\mathcal{O}_{1}-\Omega^{2}\right) \tilde{\eta}+i \Omega \mathcal{O}_{2} \tilde{\eta}=0
$$

Let us now separate variables as

$$
\eta(t, \rho)=\beta(\rho) e^{-i \omega t}, \quad \tilde{\eta}(t, \rho)=\tilde{\beta}(\rho) e^{-i \omega t},
$$

for some frequency $\omega$. Then, remarkably, one can find the following general solutions

$$
\begin{aligned}
& \eta(t, \rho)=e^{-i \omega t} e^{i \frac{\omega-\Omega}{\rho}}\left[A+B e^{i \frac{\omega-\Omega}{\rho_{c}}\left(\arctan \left(\frac{\rho}{\rho_{c}}\right)+\frac{1}{2} \log \frac{\rho-\rho_{c}}{\rho+\rho_{c}}\right)}\right], \\
& \tilde{\eta}(t, \rho)=e^{-i \omega t} e^{i \frac{\omega+\Omega}{\rho}}\left[\tilde{A}+\tilde{B} e^{i \frac{\omega+\Omega}{\rho_{c}}\left(\arctan \left(\frac{\rho}{\rho_{c}}\right)+\frac{1}{2} \log \frac{\rho-\rho_{c}}{\rho+\rho_{c}}\right)}\right],
\end{aligned}
$$

where $A, B, \tilde{A}$ and $\tilde{B}$ are complex constants which are determined by imposing boundary conditions both at the IR and UV. First of all, let us look at the regularity conditions at the pseudo-horizon $\rho=\rho_{*}$. These conditions are better studied by using the tortoise coordinates $\left(\tau, \rho_{*}\right)$, related to $(t, \rho)$ in this massless case by the following differential relations

$$
d \rho_{*}=\frac{\rho^{2}}{\rho^{4}-\rho_{c}^{4}} d \rho, \quad d \tau=d t-\frac{\rho_{c}^{4}}{\rho^{2}\left(\rho^{4}-\rho_{c}^{4}\right)} d \rho,
$$

which can be integrated as

$$
\begin{aligned}
\rho_{*} & =\frac{1}{2 \rho_{c}}\left[\arctan \left(\frac{\rho}{\rho_{c}}\right)+\frac{1}{2} \log \frac{\rho-\rho_{c}}{\rho+\rho_{c}}-\frac{\pi}{2}\right], \\
\tau & =t-\frac{1}{\rho}-\frac{1}{2 \rho_{c}}\left[\arctan \left(\frac{\rho}{\rho_{c}}\right)+\frac{1}{2} \log \frac{\rho-\rho_{c}}{\rho+\rho_{c}}-\frac{\pi}{2}\right] .
\end{aligned}
$$

Notice that the second of these equations can be simply rewritten as

$$
\tau=t-\frac{1}{\rho}-\rho_{*} .
$$


The new radial coordinate $\rho_{*}$ varies from $\rho_{*}=-\infty$ at the pseudohorizon to $\rho_{*}=0$ at the UV boundary. Actually, one can prove that in these regions it can be related to $\rho$ as

$$
\rho_{*}=-\frac{1}{\rho}+\mathcal{O}\left(\rho^{-5}\right), \quad(\rho \rightarrow \infty), \quad \rho_{*} \sim \frac{1}{4 \rho_{c}} \log \left(\rho-\rho_{c}\right), \quad\left(\rho \rightarrow \rho_{c}\right) .
$$

Inspecting the expression of $\eta$ in (D.6) one easily demonstrates that, in terms of the tortoise variable, it can be simply written as

$$
\eta\left(\tau, \rho_{*}\right)=e^{-i \frac{\Omega}{\rho}}\left[A e^{-i \omega\left(\tau+\rho_{*}\right)}+B_{*} e^{-2 i \Omega \rho_{*}} e^{-i \omega\left(\tau-\rho_{*}\right)}\right]
$$

where $B_{*}$ is a new constant, related to $B$ as $B_{*}=\exp \left[\frac{i \pi(\omega-\Omega)}{2 \rho_{c}}\right] B$. It is now clear that $\eta\left(\tau, \rho_{*}\right)$ is the superposition of ingoing and outgoing waves at the pseudohorizon. The infalling regularity condition requires that $B_{*}$ (and thus $B$ ) vanishes. Then, writing $\eta$ in our original $(t, \rho)$ coordinates, we have

$$
\eta(t, \rho)=A e^{i \frac{\omega-\Omega}{\rho}} e^{-i \omega t} .
$$

We can proceed similarly with $\tilde{\eta}$ and conclude that we should require that $\tilde{B}=0$. Therefore

$$
\tilde{\eta}(t, \rho)=\tilde{A} e^{i \frac{\omega+\Omega}{\rho}} e^{-i \omega t} .
$$

Therefore, we obtain that the fluctuations $\delta c_{x}$ and $\delta c_{y}$ regular at the pseudohorizon are

$$
\delta c_{x}(t, \rho)=\frac{1}{2}\left[A e^{i \frac{\omega-\Omega}{\rho}}+\tilde{A} e^{i \frac{\omega+\Omega}{\rho}}\right] e^{-i \omega t}, \quad \delta c_{y}(t, \rho)=\frac{1}{2 i}\left[A e^{i \frac{\omega-\Omega}{\rho}}-\tilde{A} e^{i \frac{\omega+\Omega}{\rho}}\right] e^{-i \omega t},
$$

Let us now impose the boundary conditions at the UV. These conditions are those written in (6.17) and can be fulfilled if we add two solutions of the form (D.14) with frequencies $\omega_{+}=\omega+\Omega$ and $\omega_{-}=\omega-\Omega$ with amplitudes $\vec{\beta}_{ \pm}^{(0)}$ at the UV given by (6.23), which can be explictely written as follows

$$
\vec{\beta}_{+}^{(0)}=\left(\begin{array}{c}
\frac{\epsilon_{x}+i \epsilon_{y}}{2 i \omega} \\
-\frac{\epsilon_{x}+i \epsilon_{y}}{2 \omega}
\end{array}\right), \quad \vec{\beta}_{-}^{(0)}=\left(\begin{array}{c}
\frac{\epsilon_{x}-i \epsilon_{y}}{2 i \omega} \\
\frac{\epsilon_{x}-i \epsilon_{y}}{2 \omega}
\end{array}\right) .
$$

Let $A_{ \pm}$and $\tilde{A}_{ \pm}$denote the constants in (D.14) for the frequency $\omega_{ \pm}$. From the leading UV term of our solution, we get that

$$
\vec{\beta}_{ \pm}^{(0)}=\left(\begin{array}{l}
\frac{A_{ \pm}+\tilde{A}_{ \pm}}{2} \\
\frac{A_{ \pm}-\tilde{A}_{ \pm}}{2 i}
\end{array}\right) .
$$

Let us now compare the expressions of $\vec{\beta}_{+}^{(0)}$ in (D.16) and (D.15). They only match if the constants $A_{+}$and $\tilde{A}_{+}$are given by

$$
A_{+}=-\frac{i}{\omega}\left(\epsilon_{x}+i \epsilon_{y}\right), \quad \tilde{A}_{+}=0 .
$$


By expanding around $\rho=\infty$ in (D.14) we can now obtain the amplitude $\vec{\beta}_{+}^{(1)}$ of the subleading term

$$
\beta_{+, x}^{(1)}=\frac{i}{2}\left(\omega_{+}-\Omega\right) A_{+}=\frac{i}{2} \omega A_{+}, \quad \beta_{+, y}^{(1)}=\frac{1}{2}\left(\omega_{+}-\Omega\right) A_{+}=\frac{1}{2} \omega A_{+} .
$$

Using (D.17) we can obtain $\vec{\beta}_{+, x}^{(1)}$ in terms of $\vec{\epsilon}$

$$
\vec{\beta}_{+}^{(1)}=\mathbf{M}_{+} \vec{\epsilon},
$$

where $\mathbf{M}_{+}$is the matrix defined in (6.16). Thus, the matrix $\mathbf{X}_{+}$introduced in (6.25) in this massless case is given by

$$
\mathbf{X}_{+}=i \omega \mathbf{I}
$$

Proceeding similarly for the frequency $\omega_{-}$, we obtain that the constants $A_{-}$and $\tilde{A}_{-}$must be

$$
A_{-}=0, \quad \tilde{A}_{-}=-\frac{i}{\omega}\left(\epsilon_{x}-i \epsilon_{y}\right),
$$

and the corresponding amplitude for the subleading term takes the form

$$
\beta_{-, x}^{(1)}=\frac{i}{2}\left(\omega_{-}+\Omega\right) \tilde{A}_{-}=\frac{i}{2} \omega \tilde{A}_{-}, \quad \beta_{-, y}^{(1)}=-\frac{1}{2}\left(\omega_{-}+\Omega\right) \tilde{A}_{-}=-\frac{1}{2} \omega \tilde{A}_{-},
$$

which, after using (D.21), can be related to $\vec{\epsilon}$ as

$$
\vec{\beta}_{-}^{(1)}=\mathbf{M}_{-} \vec{\epsilon} .
$$

Comparing (6.25) and (D.23) we immediately conclude that

$$
\mathbf{X}_{-}=i \omega \mathbf{I} \text {. }
$$

Using these values of $\mathbf{X}_{+}$and $\mathbf{X}_{-}$we can now obtain the conductivities from (6.27). As

$$
\begin{aligned}
& \mathbf{M}_{+} \mathbf{X}_{+} \mathbf{M}_{+}+\mathbf{M}_{-} \mathbf{X}_{-} \mathbf{M}_{-}=i \omega\left[\mathbf{M}_{+}^{2}+\mathbf{M}_{-}^{2}\right]=i \omega\left[\mathbf{M}_{+}+\mathbf{M}_{-}\right]=i \omega \mathbf{I} \\
& \mathbf{M}_{\mp} \mathbf{X}_{ \pm} \mathbf{M}_{ \pm}=i \omega \mathbf{M}_{\mp} \mathbf{M}_{ \pm}=0 .
\end{aligned}
$$

This can be summarized as follows:

$$
\boldsymbol{\sigma}(\omega)=\mathbf{I}, \quad \boldsymbol{\sigma}^{+}(\omega)=\boldsymbol{\sigma}^{-}(\omega)=0 .
$$

\section{E More on optical conductivities}

In figure 17 we show some plots for the heterodyning optical conductivities $\boldsymbol{\sigma}^{ \pm}(\omega)$. As proven in [26], a set of non-trivial relations among the components of $\boldsymbol{\sigma}$ and $\boldsymbol{\sigma}^{ \pm}$allows to take as independent components $\sigma_{x x}, \sigma_{x y}$ and $\sigma_{x x}^{ \pm}$. The relation $\boldsymbol{\sigma}(-\omega)=\boldsymbol{\sigma}(\omega)^{*}$ implies that the DC conductivity will be real. For the heterodyning conductivities, in contrast, $\boldsymbol{\sigma}^{ \pm}(-\omega)=\boldsymbol{\sigma}^{\mp}(\omega)^{*}$ and, hence, the associated DC limits will be complex.

They exhibit a similar pattern of oscillations whose amplitude increases as we approach the critical point. Eventually they sharpen into peaks as well. Comparing for example the peaks in $\sigma_{x x}^{ \pm}$with those in $\sigma_{x x}$ in figure 10 it seems apparent that they share half of them each. This is apparent in the heterodyning version of figures 11 shown below in figure 18 . Namely, while $\sigma_{x x}^{-}$has only the central stable peaks, $\sigma_{x x}^{+}$has the external peaks whose average value is $\Omega$. In figure 19 we plot $\sigma_{x x}^{ \pm}$at $\omega=0$ as a function of $\Omega / m$ and $|E| / m^{2}$. 

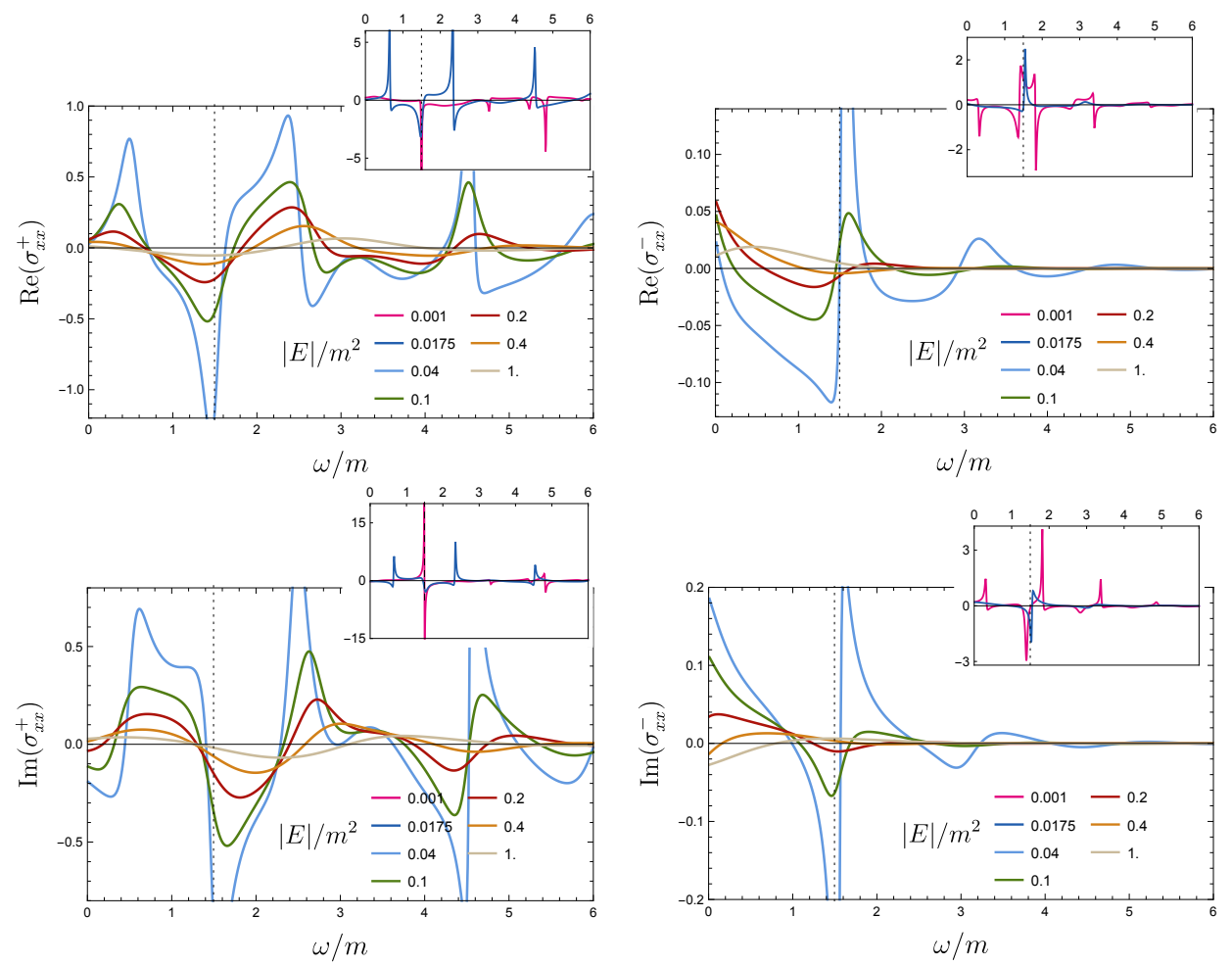

Figure 17. AC Conductivities for fixed value of $\Omega / m=1.496$ and different values of $|E| / m^{2}$. They correspond to the vertically aligned dots in figure 12 with the same color code. In the insets, the curves for low values of $|E| / m^{2} \rightarrow 0$ deep into the critical wedge, where they develop peak resonances. The value $\omega / m \rightarrow \Omega / m=1.496$ is signalled with a dashed vertical line.
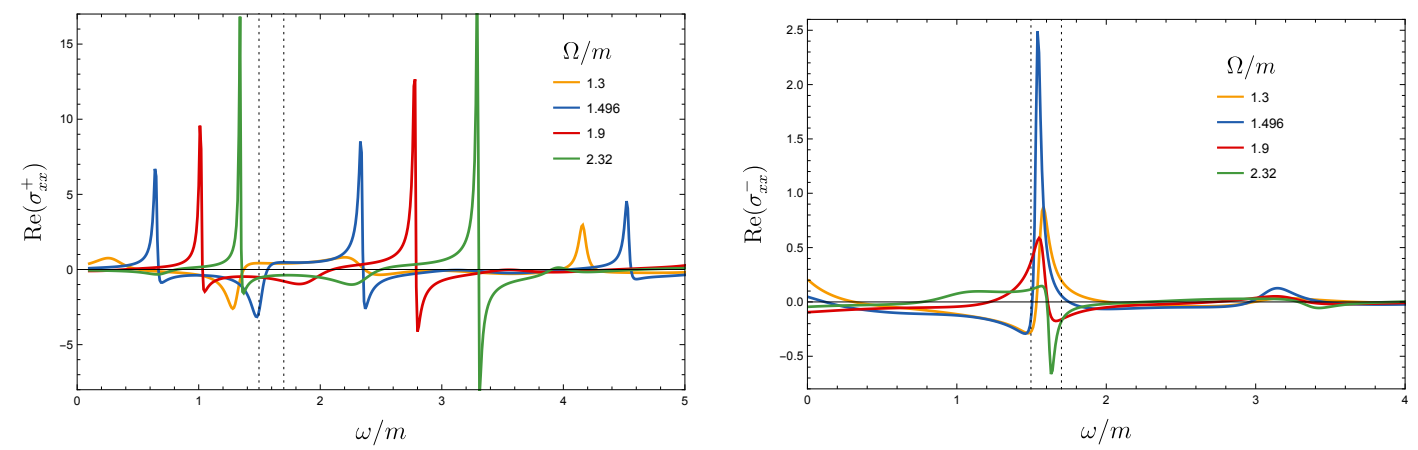

Figure 18. Peaks in the AC conductivities along the critical line to the right of the first Floquet condensate (see figure 12). The vertical dashed lines signal the critical Floquet and the meson mass frequencies respectively. The central peaks bunch in this region whereas the two lateral peaks move with $\omega$ while keeping their inter-spacing almost constant. 

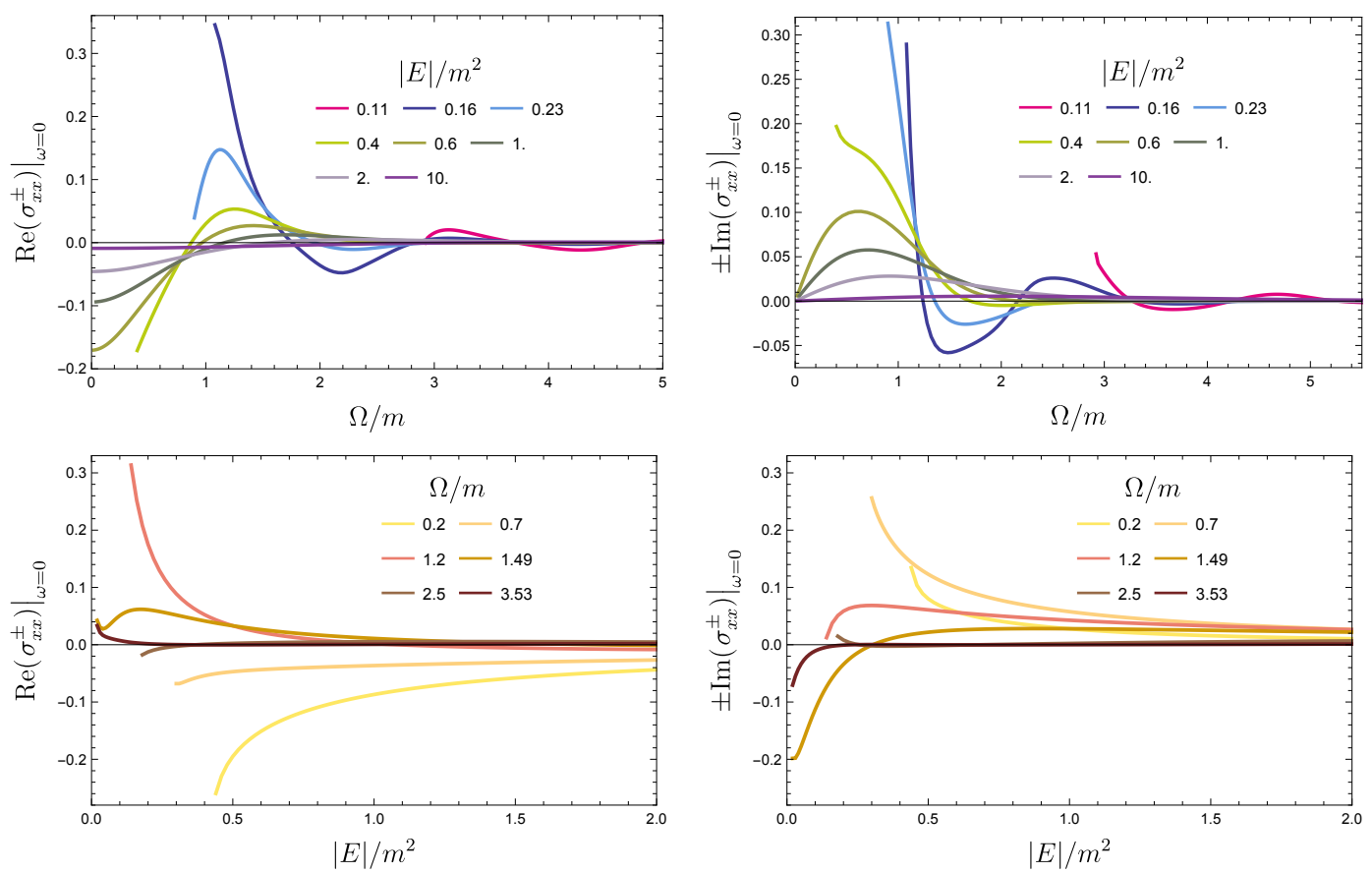

Figure 19. DC conductivities $\sigma_{x x}^{ \pm}$as function of either $\Omega / m$ or $|E| / m^{2}$.

Open Access. This article is distributed under the terms of the Creative Commons Attribution License (CC-BY 4.0), which permits any use, distribution and reproduction in any medium, provided the original author(s) and source are credited.

\section{References}

[1] M. Bukov, L. D'Alessio and A. Polkovnikov, Universal high-frequency behavior of periodically driven systems: from dynamical stabilization to Floquet engineering, Adv. Phys. 64 (2015) 139 [arXiv: 1407.4803].

[2] A. Eckardt, Colloquium: Atomic quantum gases in periodically driven optical lattices, Rev. Mod. Phys. 89 (2017) 011004 [arXiv:1606.08041] [InSPIRE].

[3] P. Weinberg, M. Bukov, L. D'Alessio, A. Polkovnikov, S. Vajna and M. Kolodrubetz, Adiabatic Perturbation Theory and Geometry of Periodically-Driven Systems, Phys. Rept. 688 (2017) 1 [arXiv: 1606.02229] [INSPIRE].

[4] M. Holthaus, Floquet engineering with quasienergy bands of periodically driven optical lattices, J. Phys. B 49 (2016) 013001 [arXiv:1510.09042].

[5] T. Oka and S. Kitamura, Floquet Engineering of Quantum Materials, Ann. Rev. Condens. Matter Phys. 10 (2019) 387 [arXiv:1804.03212].

[6] M.S. Rudner and N.H. Lindner, The Floquet Engineer's Handbook, arXiv:2003.08252 [INSPIRE].

[7] D. Fausti et al., Light-Induced Superconductivity in a Stripe-Ordered Cuprate, Science 331 (2011) 189. 
[8] M. Mitrano et al., Possible light-induced superconductivity in K3C60 at high temperature, Nature $\mathbf{5 3 0}$ (2016) 461.

[9] T. Oka and H. Aoki, Photovoltaic Hall effect in graphene, Phys. Rev. B 79 (2009) 081406 [arXiv: 0807.4767].

[10] T. Oka and H. Aoki, All optical measurement proposed for the photovoltaic Hall effect, J. Phys. Conf. Ser. 334 (2011) 012060 [arXiv: 1007.5399].

[11] T. Kitagawa, T. Oka, A. Brataas, L. Fu and E. Demler, Transport properties of non-equilibrium systems under the application of light: Photo-induced quantum Hall insulators without Landau levels, Phys. Rev. B 84 (2011) 235108 [arXiv:1104.4636].

[12] H. Deghani and M. Aditi, Optical Hall conductivity of a Floquet topological insulator, Phys. Rev. B 92 (2015) 165111 [arXiv:1506. 08687].

[13] J. Cayssol, B. Dora, F. Simon and R. Moessner, Floquet topological insulators, Phys. Status Solidi RRL 7 (2013) 101 [arXiv:1211.5623].

[14] N.H. Lindner, G. Refael and V. Galitski, Floquet Topological Insulator in Semiconductor Quantum Wells, Nat. Phys. 7 (2011) 490 [arXiv:1008.1792].

[15] P. Marini et al., Systematic study of the symmetry energy within the approach of the statistical multifragmentation model, Phys. Rev. C $8 \mathbf{8 7}$ (2013) 024603 [arXiv:1212.3126] [INSPIRE].

[16] G. Jotzu et al., Experimental realization of the topological Haldane model with ultracold fermions, Nature 515 (2014) 237 [arXiv:1406.7874] [INSPIRE].

[17] Y.H. Wang, H. Steinberg, P. Jarillo-Herrero and N. Gedik, Observation of Floquet-Bloch states on the surface of a topological insulator, Science 342 (2013) 453 [arXiv:1310.7563] [INSPIRE].

[18] X.-X. Zhang, T.T. Ong and N. Nagaosa, Theory of photoinduced Floquet Weyl semimetal phases, Phys. Rev. B 94 (2016) 235137 [arXiv:1607.05941] [InSPIRE].

[19] H. Hübener, M.A. Sentef, U. de Giovannini, A.F. Kemper and A. Rubio, Creating stable Floquet-Weyl semimetals by laser-driving of 3D Dirac materials, Nat. Commun. 8 (2017) 13940 [arXiv: 1604.03399].

[20] L. Bucciantini, S. Roy, S. Kitamura and T. Oka, Emergent Weyl nodes and Fermi arcs in a Floquet Weyl semimetal, Phys. Rev. B 96 (2017) 041126 [arXiv:1612.01541].

[21] J.M. Maldacena, The Large $N$ limit of superconformal field theories and supergravity, Int. J. Theor. Phys. 38 (1999) 1113 [hep-th/9711200] [INSPIRE].

[22] J. Casalderrey-Solana, H. Liu, D. Mateos, K. Rajagopal and U.A. Wiedemann, Gauge/String Duality, Hot QCD and Heavy Ion Collisions, Cambridge University Press, Cambridge U.K. (2014), [arXiv: 1101.0618] [INSPIRE].

[23] J. McGreevy, Holographic duality with a view toward many-body physics, Adv. High Energy Phys. 2010 (2010) 723105 [arXiv:0909.0518] [INSPIRE].

[24] A.V. Ramallo, Introduction to the AdS/CFT correspondence, Springer Proc. Phys. 161 (2015) 411 [arXiv:1310.4319] [INSPIRE].

[25] J.D. Edelstein, J.P. Shock and D. Zoakos, The AdS/CFT Correspondence and Non-perturbative QCD, AIP Conf. Proc. 1116 (2009) 265 [arXiv:0901.2534] [INSPIRE]. 
[26] K. Hashimoto, S. Kinoshita, K. Murata and T. Oka, Holographic Floquet states I: a strongly coupled Weyl semimetal, JHEP 05 (2017) 127 [arXiv:1611.03702] [INSPIRE].

[27] S. Kinoshita, K. Murata and T. Oka, Holographic Floquet states II: Floquet condensation of vector mesons in nonequilibrium phase diagram, JHEP 06 (2018) 096 [arXiv:1712.06786] [INSPIRE].

[28] O. DeWolfe, D.Z. Freedman and H. Ooguri, Holography and defect conformal field theories, Phys. Rev. D 66 (2002) 025009 [hep-th/0111135] [INSPIRE].

[29] A. Karch and E. Katz, Adding flavor to AdS/CFT, JHEP 06 (2002) 043 [hep-th/0205236] [INSPIRE].

[30] J. Erdmenger, Z. Guralnik and I. Kirsch, Four-dimensional superconformal theories with interacting boundaries or defects, Phys. Rev. D 66 (2002) 025020 [hep-th/0203020] [INSPIRE].

[31] K. Skenderis and M. Taylor, Branes in AdS and p p wave space-times, JHEP 06 (2002) 025 [hep-th/0204054] [INSPIRE].

[32] D. Arean and A.V. Ramallo, Open string modes at brane intersections, JHEP 04 (2006) 037 [hep-th/0602174] [INSPIRE].

[33] S. Takayoshi, J. Wu and T. Oka, Twisted Schwinger Effect: Pair Creation in Rotating Fields, arXiv:2005.01755 [INSPIRE].

[34] M. Kruczenski, D. Mateos, R.C. Myers and D.J. Winters, Meson spectroscopy in AdS/CFT with flavor, JHEP 07 (2003) 049 [hep-th/0304032] [INSPIRE].

[35] J. Berges, S. Borsányi and C. Wetterich, Prethermalization, Phys. Rev. Lett. 93 (2004) 142002 [hep-ph/0403234] [INSPIRE].

[36] C. Heinisch and M. Holthaus, Adiabatic preparation of Floquet condensates, J. Mod. Opt. 63 (2016) 1768 [arXiv: 1605.08199].

[37] V.P. Frolov, Merger Transitions in Brane-Black-Hole Systems: Criticality, Scaling, and Self-Similarity, Phys. Rev. D 74 (2006) 044006 [gr-qc/0604114] [InSPIRE].

[38] D. Mateos, R.C. Myers and R.M. Thomson, Holographic phase transitions with fundamental matter, Phys. Rev. Lett. 97 (2006) 091601 [hep-th/0605046] [INSPIRE].

[39] D. Mateos, R.C. Myers and R.M. Thomson, Thermodynamics of the brane, JHEP 05 (2007) 067 [hep-th/0701132] [INSPIRE].

[40] N. Seiberg and E. Witten, String theory and noncommutative geometry, JHEP 09 (1999) 032 [hep-th/9908142] [INSPIRE].

[41] C. Núñez, A. Paredes and A.V. Ramallo, Unquenched Flavor in the Gauge/Gravity Correspondence, Adv. High Energy Phys. 2010 (2010) 196714 [arXiv:1002.1088] [InSPIRE].

[42] E. Conde, H. Lin, J.M. Penín, A.V. Ramallo and D. Zoakos, D3-D5 theories with unquenched flavors, Nucl. Phys. B 914 (2017) 599 [arXiv: 1607.04998] [INSPIRE].

[43] J.M. Penín, A.V. Ramallo and D. Zoakos, Anisotropic D3-D5 black holes with unquenched flavors, JHEP 02 (2018) 139 [arXiv:1710.00548] [INSPIRE].

[44] N. Jokela, J.M. Penín, A.V. Ramallo and D. Zoakos, Gravity dual of a multilayer system, JHEP 03 (2019) 064 [arXiv: 1901.02020] [INSPIRE]. 
[45] U. Gran, N. Jokela, D. Musso, A. V. Ramallo and M. Tornsö, Holographic fundamental matter in multilayered media, JHEP 12 (2019) 038 [arXiv: 1909.01864] [INSPIRE].

[46] O. Aharony, O. Bergman, D.L. Jafferis and J. Maldacena, $N=6$ superconformal Chern-Simons-matter theories, M2-branes and their gravity duals, JHEP 10 (2008) 091 [arXiv:0806.1218] [INSPIRE].

[47] S. Hohenegger and I. Kirsch, A Note on the holography of Chern-Simons matter theories with flavour, JHEP 04 (2009) 129 [arXiv: 0903.1730] [INSPIRE].

[48] D. Gaiotto and D.L. Jafferis, Notes on adding D6 branes wrapping $R p^{3}$ in $A d S_{4} \times C P^{3}$, JHEP 11 (2012) 015 [arXiv:0903.2175] [InSPIRE].

[49] N. Jokela, J. Mas, A.V. Ramallo and D. Zoakos, Thermodynamics of the brane in Chern-Simons matter theories with flavor, JHEP 02 (2013) 144 [arXiv:1211.0630] [INSPIRE].

[50] A. Karch and E. Katz, Adding flavor to AdS/CFT, JHEP 06 (2002) 043 [hep-th/0205236] [INSPIRE].

[51] Y. Bea, N. Jokela, M. Lippert, A.V. Ramallo and D. Zoakos, Flux and Hall states in ABJM with dynamical flavors, JHEP 03 (2015) 009 [arXiv:1411.3335] [INSPIRE].

[52] C. Hoyos, T. Nishioka and A. O'Bannon, A Chiral Magnetic Effect from AdS/CFT with Flavor, JHEP 10 (2011) 084 [arXiv:1106.4030] [INSPIRE].

[53] T. Oka and L. Bucciantini, Heterodyne Hall effect in a two-dimensional electron gas, Phys. Rev. B 94 (2016) 155133.

[54] W. Kohn, Periodic thermodynamics, J. Stat. Phys. 103 (2014) 417.

[55] T. Morimoto, Y. Hatsugai and H. Aoki, Optical Hall conductivity in 2DEG and graphene QHE systems, Physica E 42 (2010) 751 [arXiv:0904.2438].

[56] Y. Ikebe, T. Morimoto, R. Masutomi, T. Okamoto, H. Aoki and R. Shimano, Optical Hall effect in the integer quantum Hall regime, Phys. Rev. Lett. 104 (2010) 256802 [arXiv: 1004.0308].

[57] Y.H. Wang, H. Steinberg, P. Jarillo-Herreo and N. Gedik, Observation of Floquet-Bloch states on the surface of a topological insulator, Science 25 (2013) 453.

[58] A. Kumar, A. Nemilentsau, K.H. Fung, G. Hanson, N.X. Fang and T. Low, Chiral plasmon in gapped Dirac systems, Phys. Rev. B 93 (2016) 041413.

[59] J.W. McIver, B.Schulte, F.-U. Stein, T. Matsuyama, G. Jotzu and A. Cavalieri, Light-induced anomalous Hall effect in graphene, Nat. Phys. 16 (2020) 38 [arXiv:1811.03522]. 